
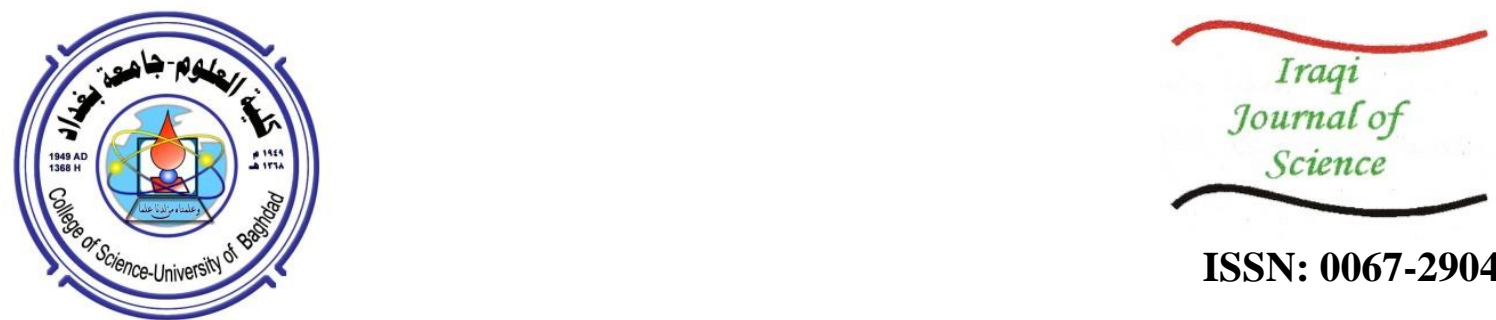

ISSN: 0067-2904

\title{
The Continuous Classical Boundary Optimal Control of Triple Nonlinear Elliptic Partial Differential Equations with State Constraints
}

\author{
Jamil A. Ali Al-Hawasy*, Nabeel A. Thyab Al-Ajeeli \\ Department of Mathematics, College of Science, Mustansiriyah University, Baghdad, Iraq \\ Received: 2/5/2020 \\ Accepted: 16/6/2020

\begin{abstract}
Our aim in this work is to study the classical continuous boundary control vector problem for triple nonlinear partial differential equations of elliptic type involving a Neumann boundary control. At first, we prove that the triple nonlinear partial differential equations of elliptic type with a given classical continuous boundary control vector have a unique "state" solution vector, by using the Minty-Browder Theorem. In addition, we prove the existence of a classical continuous boundary optimal control vector ruled by the triple nonlinear partial differential equations of elliptic type with equality and inequality constraints. We study the existence of the unique solution for the triple adjoint equations related with the triple state equations. The Fréchet derivative is obtained. Finally we prove the theorems of both the necessary and sufficient conditions for optimality of the triple nonlinear partial differential equations of elliptic type through the Kuhn-Tucker-Lagrange's Multipliers theorem with equality and inequality constraints.
\end{abstract}

Keywords: optimal control vector, triple nonlinear elliptic equations, necessary and sufficient conditions for optimality

\section{مسألة السيطرة الددودية الامثلية التقليدية المستمرة لثلاثي من المعادلات التفاضلية الجزئية الغير خطية من النوع الاهليجي بوجود قيود الحالة}

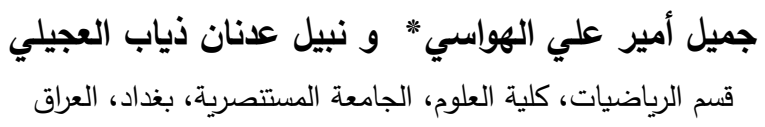

*Email: jhawassy17@uomustanriyah.edu.iq 


\section{Introduction}

In many fields, the optimal control problems play a significant role in life. Different examples of the applications of such problems are presented in medicine [1], aircraft industry [2], electric power production [3], economic growth [4], and many other fields.

All these applications pushed many investigators to a higher level of interest in studying the optimal control problem for nonlinear ordinary differential equations [5], for different types of linear partial differential equations, including the hyperbolic, parabolic and elliptic [6- 8], or for couple nonlinear partial differential equations of these three types [9-11]. While other authors [12, 13] studied these three types but included a Neumann boundary control. More recently, optimal control problems were studied for triple partial differential equations of these three types [14-16]. Also, the optimal control problem involving Neumann boundary control for triple partial differential equations of parabolic type was also recently investigated [17]. All these investigations motivated us to seek the optimal control problem, involving Neumann boundary control ruled by the triple nonlinear partial differential equations of elliptic type.

At first, our aim in this work is to prove that system of the triple nonlinear partial differential equations of elliptic type with a given classical continuous boundary control vector, which has a unique "state" solution vector, by using the Minty-Browder Theorem. Then, we prove the existence of a classical continuous boundary optimal control vector, ruled by the triple nonlinear partial differential equations of elliptic type with equality and inequality constraints.

We study the existence of the unique solution for the system of the triple adjoint equations related with the triple state equations. At the end, we prove the theorems of both the necessary and sufficient conditions for optimality of the triple nonlinear partial differential equations of elliptic type through the Kuhn-Tucker-Lagrange's Multipliers with equality and inequality constraints.

\section{Problem Description}

Let $\Psi$ be a bounded and open connected subset in $\mathrm{R}^{2}$ with Lipshitz boundary $\partial \Psi$. The optimal control problem is considered by the "state vector equation" which consists of the TNLEPDEs triple nonlinear elliptic partial differential equations with the Neumann boundary control.

$$
\begin{aligned}
& \mathrm{A}_{1} \mathrm{~b}_{1}+\mathrm{b}_{1}-\mathrm{b}_{2}-\mathrm{b}_{3}+\mathrm{m}_{1}\left(\mathrm{x}, \mathrm{b}_{1}\right)=\mathrm{K}_{1}(\mathrm{x}), \text { in } \Psi \\
& \mathrm{A}_{2} \mathrm{~b}_{2}+\mathrm{b}_{1}+\mathrm{b}_{2}+\mathrm{b}_{3}+\mathrm{m}_{2}\left(\mathrm{x}, \mathrm{b}_{2}\right)=\mathrm{F}_{2}(\mathrm{x}), \text { in } \Psi \\
& \mathrm{A}_{3} \mathrm{~b}_{3}+\mathrm{b}_{1}-\mathrm{b}_{2}+\mathrm{b}_{3}+\mathrm{m}_{3}\left(\mathrm{x}, \mathrm{b}_{3}\right)=\mathrm{K}_{3}(\mathrm{x}), \text { in } \Psi \\
& \sum_{\sigma, \mathrm{j}=1}^{2} \mathrm{a}_{1_{\sigma \mathrm{j}}} \frac{\partial \mathrm{b}_{1}}{\partial \mathrm{n}_{1}}=\mathrm{d}_{1}, \text { on } \partial \Psi \\
& \sum_{\sigma, \mathrm{j}=1}^{2} \mathrm{a}_{2 \sigma \mathrm{j}} \frac{\partial \mathrm{b}_{2}}{\partial \mathrm{n}_{2}}=\mathrm{d}_{2}, \text { on } \partial \Psi \\
& \sum_{\sigma, \mathrm{j}=1}^{2} \mathrm{a}_{3 \sigma \mathrm{j}} \frac{\partial \mathrm{b}_{3}}{\partial \mathrm{n}_{3}}=\mathrm{d}_{3}, \text { on } \partial \Psi
\end{aligned}
$$

where

$\mathrm{A}_{\mathrm{r}} \mathrm{b}_{\mathrm{r}}=-\sum_{\sigma, \mathrm{j}=1}^{2} \frac{\partial}{\partial \mathrm{x}_{\sigma}}\left(\mathrm{a}_{\mathrm{r}_{\sigma \mathrm{j}}}(\mathrm{x}) \frac{\partial \mathrm{b}_{\mathrm{r}}}{\partial \mathrm{x}_{\mathrm{j}}}\right) \quad, \mathrm{r}=1,2,3 \quad \mathrm{a}_{\mathrm{r}_{\sigma \mathrm{j}}}=\mathrm{a}_{\mathrm{r}_{\sigma \mathrm{j}}}\left(\mathrm{x}_{\sigma \mathrm{j}}\right) \in \mathrm{C}^{\infty}(\Psi) \quad, \quad$ for $\quad \sigma, \mathrm{j}=1,2$ $\left(\mathrm{d}_{1}, \mathrm{~d}_{2}, \mathrm{~d}_{3}\right)=\left(\mathrm{d}_{1}(\mathrm{x}), \mathrm{d}_{2}(\mathrm{x}), \mathrm{d}_{3}(\mathrm{x})\right) \in\left(\mathrm{L}_{2}(\partial \Psi)\right)^{3}$ is the Neumann boundary control vector. The correspond "state" solution vector to the Neumann boundary control vector is $\left(b_{1}, b_{2}, b_{3}\right)=$ $\left(b_{1}(x), b_{2}(x), b_{3}(x)\right) \in\left(H^{1}(\Psi)\right)^{3}, \quad\left(m_{1}, m_{2}, m_{3}\right)=\left(m_{1}\left(x, b_{1}\right), m_{2}\left(x, b_{2}\right), m_{3}\left(x, b_{3}\right)\right)$, $\left(5_{1}, 5_{2}, 5_{3}\right)=\left(5_{1}(x), 5_{2}(x), 5_{3}(x)\right) \in\left(L_{2}(\Psi)\right)^{3}$, which is a vector of functions.

The control constraints are

$\overrightarrow{\mathrm{d}} \in \overrightarrow{\mathrm{E}}, \overrightarrow{\mathrm{E}} \subset\left(\mathrm{L}_{2}(\partial \Psi)\right)^{3}$, where $\overrightarrow{\mathrm{d}}=\left(\mathrm{d}_{1}, \mathrm{~d}_{2}, \mathrm{~d}_{3}\right)$ and $\overrightarrow{\mathrm{E}}=\mathrm{E}_{1} \times \mathrm{E}_{2} \times \mathrm{E}_{3}$, with

$\overrightarrow{\mathrm{E}}=\overrightarrow{\mathrm{E}}_{\overrightarrow{\mathrm{D}}}=\left\{\overrightarrow{\mathrm{E}} \in\left(\mathrm{L}_{2}(\partial \Psi)\right)^{3} \mid \overrightarrow{\mathrm{E}}=\left(\mathrm{E}_{1}, \mathrm{E}_{2}, \mathrm{E}_{3}\right) \in \overrightarrow{\mathrm{D}}\right.$ a. e in $\left.\partial \Psi\right\}$,

where $\vec{D}=D_{1} \times D_{2} \times D_{3}$, with $\vec{D} \subset R^{3}$ is a convex and compact set.

The cost function and the equality and inequality constraints are given by:

$$
\begin{array}{r}
\mathrm{T}_{0}(\overrightarrow{\mathrm{d}})=\iint_{\Psi}\left[\mathrm{T}_{01}\left(\mathrm{x}, \mathrm{b}_{1}\right)+\mathrm{T}_{02}\left(\mathrm{x}, \mathrm{b}_{2}\right)+\mathrm{T}_{03}\left(\mathrm{x}, \mathrm{b}_{3}\right)\right] \mathrm{dx}_{1} \mathrm{dx}_{2} \\
+\int_{\partial \Psi}\left[\mathrm{T}_{04}\left(\mathrm{x}, \mathrm{d}_{1}\right)+\mathrm{T}_{05}\left(\mathrm{x}, \mathrm{d}_{2}\right)+\mathrm{T}_{06}\left(\mathrm{x}, \mathrm{d}_{3}\right)\right] \mathrm{d} \gamma
\end{array}
$$




$$
\begin{aligned}
& \mathrm{T}_{1}(\overrightarrow{\mathrm{d}})=\iint_{\Psi}\left[\mathrm{G}_{11}\left(\mathrm{x}, \mathrm{b}_{1}\right)+\mathrm{G}_{12}\left(\mathrm{x}, \mathrm{b}_{2}\right)+\mathrm{G}_{13}\left(\mathrm{x}, \mathrm{b}_{3}\right)\right] d \mathrm{dx}_{1} \mathrm{dx}_{2} \\
& +\int_{\partial \Psi}\left[\mathrm{G}_{14}\left(\mathrm{x}, \mathrm{d}_{1}\right)+\mathrm{t}_{15}\left(\mathrm{x}, \mathrm{d}_{2}\right)+\mathrm{t}_{16}\left(\mathrm{x}, \mathrm{d}_{3}\right)\right] \mathrm{d} \gamma=0 \\
& \mathrm{~T}_{2}(\overrightarrow{\mathrm{d}})=\iint_{\Psi}\left[\mathrm{T}_{21}\left(\mathrm{x}, \mathrm{b}_{1}\right)+\mathrm{T}_{22}\left(\mathrm{x}, \mathrm{b}_{2}\right)+\mathrm{T}_{23}\left(\mathrm{x}, \mathrm{b}_{3}\right)\right] d \mathrm{dx}_{1} \mathrm{dx}_{2} \\
& +\int_{\partial \Psi}\left[\mathrm{T}_{24}\left(\mathrm{x}, \mathrm{d}_{1}\right)+\mathrm{T}_{25}\left(\mathrm{x}, \mathrm{d}_{2}\right)+\mathrm{T}_{26}\left(\mathrm{x}, \mathrm{d}_{3}\right)\right] \mathrm{d} \gamma \leq 0
\end{aligned}
$$

The set of admissible control is

$$
\overrightarrow{\mathrm{E}}_{\mathrm{A}}=\left\{\overrightarrow{\mathrm{d}} \in \overrightarrow{\mathrm{E}} \mid \mathrm{G}_{1}(\overrightarrow{\mathrm{d}})=0, \mathrm{~T}_{2}(\overrightarrow{\mathrm{d}}) \leq 0\right\}
$$

The classical continuous boundary control vector problem is to minimize (7) subject to the state constraints (8) and (9), i.e. to find $\vec{d}$, such that

$\overrightarrow{\mathrm{d}} \in \overrightarrow{\mathrm{E}}_{\mathrm{A}}$ and $\mathrm{G}_{0}(\overrightarrow{\mathrm{d}})=\min _{\overrightarrow{\mathrm{e}} \in \overrightarrow{\mathrm{E}}} \mathrm{G}_{0}(\overrightarrow{\mathrm{e}})$.

Let $\overrightarrow{\mathrm{T}}=(\mathrm{T})^{3}=\left(\mathrm{H}^{1}(\Psi)\right)^{3}$, the notations $(\mathrm{t}, \mathrm{t}) \_\left(\mathrm{L} \_2(\Psi)\right)$, and $\|\mathrm{T}\|\left(\mathrm{L} \_2(\Psi)\right)\left(\|\mathrm{T}\| \_\left(\mathrm{L} \_2(\partial \Psi)\right)\right)$

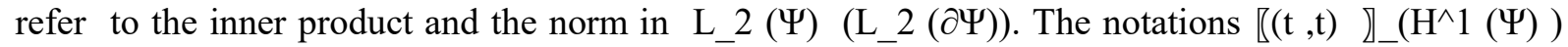
and $\|T\|\left(H^{\wedge} 1(\Psi)\right)$ refer to the inner product and the norm in $\mathrm{H}^{\wedge} 1(\Psi)$, the notations $\left(\mathrm{t}^{\overrightarrow{ }}, \mathrm{t} \overrightarrow{\mathrm{s}}\right.$

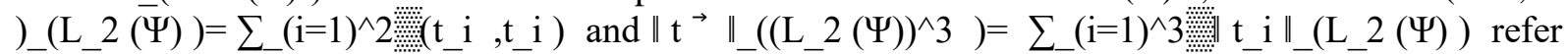
to the inner product and the norm in $\left(\mathrm{L}_{-} 2(\bar{\Psi})\right)^{\wedge} \overline{3}$, while the notations $\left(\mathrm{t}^{-}{ }^{-}, \mathrm{t}^{-}\right)_{-}\left(\mathrm{L}_{-} 2(\Psi)\right)=$ $\sum(\mathrm{i}=1)^{\wedge} 3$ 3. product and the norm in $\overrightarrow{\mathrm{T}} \overrightarrow{\text {, finally }} \mathrm{T} \vec{\wedge} *$ is referred to the dual of $\mathrm{T} \overrightarrow{\text {. }}$

\section{Weak formulation of the triple state equations}

To find the weak formulation of problem (1-6), let

$$
\begin{aligned}
\overrightarrow{\mathrm{T}} & =\mathrm{T}_{1} \times \mathrm{T}_{2} \times \mathrm{T}_{3}=\mathrm{H}^{1}(\Psi) \times \mathrm{H}^{1}(\Psi) \times \mathrm{H}^{1}(\Psi) \\
& =\left\{\overrightarrow{\mathrm{t}}: \overrightarrow{\mathrm{t}}=\left(\mathrm{t}_{1}, \mathrm{t}_{2}, \mathrm{t}_{3}\right) \in\left(\mathrm{H}^{1}(\Psi)\right)^{3}, \text { with } \mathrm{t}_{1}, \mathrm{t}_{2}, \mathrm{t}_{3} \text { satisfy (4)-(6), respectively on } \partial \Psi\right\} .
\end{aligned}
$$

By multiplying both sides of equations (1),(2) and (3) by $t_{1} \in T_{1}, t_{2} \in T_{2} t_{3} \in T_{3}$, respectively, integrating both sides of each one of the obtained equations with respect to $x$, and then using the generalize Green's theorem, we get

$$
\begin{array}{r}
\mathrm{a}_{1}\left(\mathrm{~b}_{1}, \mathrm{t}_{1}\right)-\left(\mathrm{b}_{2}+\mathrm{b}_{3}, \mathrm{t}_{1}\right)_{\mathrm{L}_{2}(\Psi)}+\left(\mathrm{m}_{1}\left(\mathrm{x}, \mathrm{b}_{1}\right), \mathrm{t}_{1}\right)_{\mathrm{L}_{2}(\Psi)} \\
=\left(\mathrm{s}_{1}(\mathrm{x}), \mathrm{t}_{1}\right)_{\mathrm{L}_{2}(\Psi)}+\left(\mathrm{d}_{1}, \mathrm{t}_{1}\right)_{\mathrm{L}_{2}(\partial \Psi)}, \forall \mathrm{t}_{1} \in \mathrm{T}_{1} \\
\mathrm{a}_{2}\left(\mathrm{~b}_{2}, \mathrm{t}_{2}\right)+\left(\mathrm{b}_{1}+\mathrm{b}_{3}, \mathrm{t}_{2}\right)_{\mathrm{L}_{2}(\Psi)}+\left(\mathrm{m}_{2}\left(\mathrm{x}, \mathrm{b}_{2}\right), \mathrm{t}_{2}\right)_{\mathrm{L}_{2}(\Psi)} \\
=\left(\mathrm{s}_{2}(\mathrm{x}), \mathrm{t}_{2}\right)_{\mathrm{L}_{2}(\Psi)}+\left(\mathrm{d}_{2}, \mathrm{t}_{2}\right)_{\mathrm{L}_{2}(\partial \Psi)}, \forall \mathrm{t}_{2} \in \mathrm{T}_{2}
\end{array}
$$

and

$$
\begin{aligned}
\mathrm{a}_{3}\left(\mathrm{~b}_{3}, \mathrm{t}_{3}\right)+\left(\mathrm{b}_{1}-\mathrm{b}_{2}, \mathrm{t}_{3}\right)_{\mathrm{L}_{2}(\Psi)}+\left(\mathrm{m}_{3}\left(\mathrm{x}, \mathrm{b}_{3}\right), \mathrm{t}_{3}\right)_{\mathrm{L}_{2}(\Psi)} \\
\quad=\left(\mathrm{s}_{3}(\mathrm{x}), \mathrm{t}_{3}\right)_{\mathrm{L}_{2}(\Psi)}+\left(\mathrm{d}_{3}, \mathrm{t}_{3}\right)_{\mathrm{L}_{2}(\partial \Psi)}, \forall \mathrm{t}_{3} \in \mathrm{T}_{3}
\end{aligned}
$$

By adding equations (11), (12) and (13), we get

$$
\begin{aligned}
\mathrm{a}(\overrightarrow{\mathrm{b}}, \overrightarrow{\mathrm{t}})+\left(\mathrm{m}_{1}\left(\mathrm{x}, \mathrm{b}_{1}\right), \mathrm{t}_{1}\right)_{\mathrm{L}_{2}(\Psi)}+\left(\mathrm{m}_{2}\left(\mathrm{x}, \mathrm{b}_{2}\right), \mathrm{t}_{2}\right)_{\mathrm{L}_{2}(\Psi)}+\left(\mathrm{m}_{3}\left(\mathrm{x}, \mathrm{b}_{3}\right), \mathrm{t}_{3}\right)_{\mathrm{L}_{2}(\Psi)} \\
=\left(\mathrm{s}_{1}(\mathrm{x}), \mathrm{t}_{1}\right)_{\mathrm{L}_{2}(\Psi)}+\left(\mathrm{d}_{1}, \mathrm{t}_{3}\right)_{\mathrm{L}_{2}(\partial \Psi)}+\left(\mathrm{s}_{2}(\mathrm{x}), \mathrm{t}_{2}\right)_{\mathrm{L}_{2}(\Psi)}+\left(\mathrm{d}_{2}, \mathrm{t}_{2}\right)_{\mathrm{L}_{2}(\partial \Psi)} \\
+\left(\mathrm{s}_{3}(\mathrm{x}), \mathrm{t}_{3}\right)_{\mathrm{L}_{2}(\Psi)}+\left(\mathrm{d}_{3}, \mathrm{t}_{3}\right)_{\mathrm{L}_{2}(\partial \Psi)}, \forall\left(\mathrm{t}_{1}, \mathrm{t}_{2}, \mathrm{t}_{3}\right) \in \mathrm{T}
\end{aligned}
$$

where

$$
\begin{aligned}
a(\vec{b}, \vec{t})= & a_{1}\left(b_{1}, t_{1}\right)-\left(b_{2}+b_{3}, t_{1}\right)_{L_{2}(\Psi)}+a_{2}\left(b_{2}, t_{2}\right)+\left(b_{1}+b_{3}, t_{2}\right)_{L_{2}(\Psi)} \\
& +a_{3}\left(b_{3}, t_{3}\right)+\left(b_{1}+b_{2}, t_{3}\right)_{L_{2}(\Psi)}
\end{aligned}
$$

with

$\mathrm{a}_{\mathrm{r}}\left(\mathrm{b}_{\mathrm{r}}, \mathrm{t}_{\mathrm{r}}\right)=\int_{\Psi}\left(\sum_{\mathrm{i}, \mathrm{j}=1}^{2} \mathrm{a}_{\mathrm{r}_{\mathrm{ij}}} \frac{\partial \mathrm{b}_{\mathrm{r}}}{\partial \mathrm{x}_{\mathrm{i}}} \frac{\partial \mathrm{t}_{\mathrm{r}}}{\partial \mathrm{x}_{\mathrm{j}}}+\mathrm{b}_{\mathrm{r}} \mathrm{t}_{\mathrm{r}}\right) \mathrm{dx}$

which satisfies

$a_{r}\left(b_{r}, t_{r}\right) \geq c_{1 r}\left\|b_{r}\right\|_{H^{1}(\Psi)}^{2}$, where $c_{1 r} \geq 0, r=1,2,3$

$\left|\mathrm{a}_{\mathrm{r}}\left(\mathrm{b}_{\mathrm{r}}, \mathrm{t}_{\mathrm{r}}\right)\right| \leq \mathrm{c}_{2 \mathrm{r}}\left\|\mathrm{b}_{\mathrm{r}}\right\|_{\mathrm{H}^{1}(\Psi)}^{2}\left\|\mathrm{~T}_{\mathrm{r}}\right\|_{\mathrm{H}^{1}(\Psi)}^{2}$, where $c_{2 \mathrm{r}} \geq 0, \mathrm{r}=1,2,3$.

The following assumptions are useful to prove the existence theorem of a unique solution of the weak form (14).

\section{Assumption (I):}

a) $\quad \mathrm{a}(\overrightarrow{\mathrm{b}}, \overrightarrow{\mathrm{t}})$ is coercive, i.e $\frac{\mathrm{a}(\overrightarrow{\mathrm{b}}, \overrightarrow{\mathrm{b}})}{\|\overrightarrow{\mathrm{b}}\|_{\left(\mathrm{H}^{1}(\Psi)\right)^{3}}} \geq \mathrm{c}\|\overrightarrow{\mathrm{b}}\|_{\left(\mathrm{H}^{1}(\Psi)\right)^{3}}>0, \forall \overrightarrow{\mathrm{b}} \in \overrightarrow{\mathrm{T}}$ 
b) $\quad a(\vec{b}, \vec{t})$ is continuous, i.e.

$$
|\mathrm{a}(\overrightarrow{\mathrm{b}}, \overrightarrow{\mathrm{t}})| \leq \ell 1\|\overrightarrow{\mathrm{b}}\|_{\left(\mathrm{H}^{1}(\Psi)\right)^{3}}\|\overrightarrow{\mathrm{t}}\|_{\left(\mathrm{H}^{1}(\Psi)\right)^{3}}, \ell 1>0, \forall \overrightarrow{\mathrm{b}}, \overrightarrow{\mathrm{t}} \in \overrightarrow{\mathrm{T}}
$$

c) $\mathrm{m}_{1}, \mathrm{~m}_{2}$ and $\mathrm{m}_{3}$ are of Carathéodory type on $\Psi \times \mathbb{R}$ and the following sub linearity conditions with respect to $b_{1}, b_{2}, b_{3}$ are satisfied, respectively, i.e.

$$
\left|\mathrm{m}_{\sigma}\left(\mathrm{x}, \mathrm{b}_{\sigma}\right)\right| \leq \phi_{\sigma}(\mathrm{x})+\overline{\mathrm{c}}_{\sigma}\left|\mathrm{b}_{\sigma}\right|, \forall\left(\mathrm{x}, \mathrm{b}_{\sigma}\right) \in \Psi \times \mathbb{R} \text { with } \phi_{\sigma} \in \mathrm{L}_{2}(\Psi), \overline{\mathrm{c}}_{\mathrm{i}}>0 \text { for } \sigma=
$$

d) $\mathrm{m}_{\sigma}\left(\mathrm{x}, \mathrm{b}_{\sigma}\right)$ are monotone with respect to $\mathrm{b}_{\sigma}$ for each $\mathrm{x} \in \Psi$, and $\mathrm{m}_{\sigma}(\mathrm{x}, 0)=0, \forall \mathrm{x} \in \Psi$, $\sigma=1,2,3$.

e) $\quad \zeta_{\sigma}(\mathrm{x})$ are of the Carathéodory type on $\Psi$ and satisfy $\left|\zeta_{\sigma}(\mathrm{x})\right| \leq \phi \mathrm{j}(\mathrm{x}), \forall \mathrm{x} \in \Psi$, with $\phi \mathrm{j}(\mathrm{x}) \in$ $\mathrm{L}_{2}(\Psi), \sigma, \mathrm{j}=1,2,3$

Theorem (1): If assumption (I) is hold, and if one of the functions $m_{1}, m_{2}$ or $m_{3}$ in (14) is strictly monotone, then for each fixed classical continuous boundary optimal control vector $\overrightarrow{\mathrm{d}} \in \overrightarrow{\mathrm{E}}_{\mathrm{A}}$, the weak form of (14) has a unique "state" solution vector $\vec{b} \in \vec{T}$.

Proof: It is clear that the existence of a unique solution of (14) is obtained after the usage of assumptions (I), then theorem (1) in reference [18] is applied.

\section{Existence of the Classical Continuous Boundary Optimal Control Vector}

In this section, the theorem of the existence of a classical continuous boundary optimal control vector under the suitable assumptions is proved. However, before proving it, it is necessary to deal with the following lemmas and assumptions.

Lemma (1): If the assumption (I) is hold, the functions $\mathrm{m}_{1}, \mathrm{~m}_{2}, \mathrm{~m}_{3}$ are Lipschitz continuous with respect to $b_{1}, b_{2}, b_{3}$, res respectively, and if $5_{1}(x), 5_{2}(x), 5_{3}(x)$ are bounded, then the mapping $\overrightarrow{\mathrm{d}} \rightarrow \overrightarrow{\mathrm{b}}_{\overrightarrow{\mathrm{d}}}$ is Lipschitz continuous from $\overrightarrow{\mathrm{E}}_{\overrightarrow{\mathrm{D}}}$ into $\left(\mathrm{L}_{2}(\Psi)\right)^{3}$, i.e.

$\|\overrightarrow{\Delta \mathrm{b}}\|_{\left(\mathrm{L}_{2}(\Psi)\right)^{3}} \leq \mathrm{L}\|\overrightarrow{\Delta \mathrm{d}}\|_{\left(\mathrm{L}_{2}(\partial \Psi)\right)^{3}}$, with $\mathrm{L}>0$.

Proof: Assume that $\overrightarrow{\mathrm{d}}, \overrightarrow{\mathrm{d}}^{\prime} \in \overrightarrow{\mathrm{E}}$ are two given controls, then there corresponding "state" solution vectors (of the weak form (14)) are $\vec{b}, \vec{b}^{\prime}$. By subtracting the above three obtained weak forms from their corresponding ones in (14), putting $\overrightarrow{\Delta b}=\vec{b}^{\prime}-\vec{b}$ and $\overrightarrow{\Delta d}=\vec{d}^{\prime}-\vec{d}$, with $\vec{t}=\overrightarrow{\Delta b}$, then adding the obtained three equations, we get

$$
\begin{gathered}
\mathrm{a}_{1}\left(\Delta \mathrm{b}_{1}, \Delta \mathrm{b}_{1}\right)+\mathrm{a}_{2}\left(\Delta \mathrm{b}_{2}, \Delta \mathrm{b}_{2}\right)+\mathrm{a}_{3}\left(\Delta \mathrm{b}_{3}, \Delta \mathrm{b}_{3}\right)+\left(\mathrm{m}_{1}\left(\mathrm{x}, \mathrm{b}_{1}+\Delta \mathrm{b}_{1}\right)-\mathrm{m}_{1}\left(\mathrm{x}, \mathrm{b}_{1}\right), \Delta \mathrm{b}_{1}\right)_{\mathrm{L}_{2}(\Psi)} \\
+\left(\mathrm{m}_{2}\left(\mathrm{x}, \mathrm{b}_{2}+\Delta \mathrm{b}_{2}\right)-\mathrm{m}_{2}\left(\mathrm{x}, \mathrm{b}_{2}\right), \Delta \mathrm{b}_{2}\right)_{\mathrm{L}_{2}(\Psi)}+\left(\mathrm{m}_{3}\left(\mathrm{x}, \mathrm{b}_{3}+\Delta \mathrm{b}_{3}\right)-\mathrm{m}_{3}\left(\mathrm{x}, \mathrm{b}_{3}\right), \Delta \mathrm{b}_{3}\right)_{\mathrm{L}_{2}(\Psi)} \\
=\left(\Delta \mathrm{d}_{1}, \Delta \mathrm{b}_{1}\right)_{\mathrm{L}_{2}(\partial \Psi)}+\left(\Delta \mathrm{d}_{2}, \Delta \mathrm{b}_{2}\right)_{\mathrm{L}_{2}(\partial \Psi)}+\left(\Delta \mathrm{d}_{3}, \Delta \mathrm{b}_{3}\right)_{\mathrm{L}_{2}(\partial \Psi)}
\end{gathered}
$$

By using assumption A-(a,d), taking the absolute value for both sides of (16), it becomes

$$
\begin{aligned}
c\|\overrightarrow{\Delta \mathrm{b}}\|_{\left(\mathrm{H}^{1}(\Psi)\right)^{3}}^{2} & \leq \theta_{1}\left\|\Delta \mathrm{b}_{1}\right\|_{\mathrm{H}^{1}(\Psi)}^{2}+\theta_{2}\left\|\Delta \mathrm{b}_{2}\right\|_{\mathrm{H}^{1}(\Psi)}^{2}+\theta_{3}\left\|\Delta \mathrm{b}_{3}\right\|_{\mathrm{H}^{1}(\Psi)}^{2} \\
& \leq\left|\left(\Delta \mathrm{d}_{1}, \Delta \mathrm{b}_{1}\right)_{\mathrm{L}_{2}(\partial \Psi)}\right|+\left|\left(\Delta \mathrm{d}_{2}, \Delta \mathrm{b}_{2}\right)_{\mathrm{L}_{2}(\partial \Psi)}\right|+\left|\left(\Delta \mathrm{d}_{3}, \Delta \mathrm{b}_{3}\right)_{\mathrm{L}_{2}(\partial \Psi)}\right|
\end{aligned}
$$

By using the Cauchy-Schwarz inequality and then the trace operator in the right side, on (17), we obtain

$$
\begin{gathered}
\mathrm{c}\|\overrightarrow{\Delta \mathrm{b}}\|_{\left(\mathrm{H}^{1}(\Psi)\right)^{3}}^{2} \leq 3 \mathrm{c}_{1}\|\overrightarrow{\Delta \mathrm{d}}\|_{\left(\mathrm{L}_{2}(\partial \Psi)\right)^{3}}+\|\overrightarrow{\Delta \overrightarrow{\mathrm{b}}}\|_{\left(\mathrm{H}^{1}(\Psi)\right)^{3}} \Rightarrow \\
\|\overrightarrow{\Delta \mathrm{b}}\|_{\left(\mathrm{H}^{1}(\Psi)\right)^{3}} \leq \mathrm{L}^{2}\|\overrightarrow{\Delta \mathrm{d}}\|_{\left(\mathrm{L}_{2}(\partial \Psi)\right)^{3}}, \text { where } \mathrm{L}^{2}=\frac{3 \mathrm{c}_{1}}{c}
\end{gathered}
$$

which gives

$$
\|\overrightarrow{\Delta \mathrm{b}}\|_{\left(\mathrm{L}_{2}(\Psi)\right)^{3}} \leq \mathrm{L}\|\overrightarrow{\Delta \mathrm{d}}\|_{\left(\mathrm{L}_{2}(\partial \Psi)\right)^{3}}
$$

\section{Assumption (II):}

Assume that $\mathrm{G} P \mathrm{P} 1$, $\mathrm{G} \_\mathrm{P} 2$, $\mathrm{G} \_\mathrm{P} 3$ on $\Psi \times \mathrm{R}$ and $\mathrm{G}+\mathrm{P} 4$, $\mathrm{G} \_\mathrm{P} 5$, $\mathrm{G} \_\mathrm{P} 6$ on $\Psi \times \mathrm{D}$ are of the Carathéodory type, then the following are satisfied for each $\mathrm{P}=0,1,2$ :

$\left|\mathrm{G} \_\mathrm{P} 1\left(\mathrm{x}, \mathrm{b} \_1\right)\right| \leq \mathrm{Y} \_\left(\mathrm{P} \_1\right)(\mathrm{x})+\mathrm{c} \_\mathrm{P} 1 \mathrm{~b} \_1 \wedge 2,\left|\mathrm{G} \_\mathrm{P} 2\left(\mathrm{x}, \mathrm{b} \_2\right)\right| \leq \mathrm{Y} \_\left(\mathrm{P} \_2\right)(\mathrm{x})+\mathrm{c} \_\mathrm{P} 2$ b_2^2,

$\left|\mathrm{G}_{\mathrm{P} 3}\left(\mathrm{x}, \mathrm{b}_{3}\right)\right| \leq \gamma_{\mathrm{P}_{3}}(\mathrm{x})+c_{\mathrm{P} 3} \mathrm{~b}_{3}^{2},\left|\mathrm{G}_{\mathrm{P} 4}\left(\mathrm{x}, \mathrm{d}_{1}\right)\right| \leq \Upsilon_{\mathrm{P}_{4}}(\mathrm{x})+c_{\mathrm{P} 4} \mathrm{~d}_{1}^{\overline{2}}$,

$\left|\mathrm{G}_{\mathrm{P} 5}\left(\mathrm{x}, \mathrm{d}_{2}\right)\right| \leq r_{\mathrm{P}_{5}}(\mathrm{x})+c_{\mathrm{P} 5} \mathrm{~d}_{2}^{2}$, and $\left|\mathrm{G}_{\mathrm{P} 6}\left(\mathrm{x}, \mathrm{d}_{3}\right)\right| \leq r_{\mathrm{P}_{6}}(\mathrm{x})+c_{\mathrm{P} 6} \mathrm{~d}_{3}^{2}$, 
where $Y_{\mathrm{P}_{1}}, \Upsilon_{\mathrm{P}_{2}}, \Upsilon_{\mathrm{P}_{3}} \in \mathrm{L}_{1}(\Psi), \Upsilon_{\mathrm{P}_{4}}, \Upsilon_{\mathrm{P}_{5}}, \Upsilon_{\mathrm{P}_{6}} \in \mathrm{L}_{1}(\partial \Psi)$ and $c_{\mathrm{P}_{\sigma}} \geq 0$ for $\sigma=1,2,3,4,5,6$.

Lemma (2): If assumption (II) is held, then the functional $\mathrm{G}_{-} \mathrm{P}\left(\mathrm{d}^{\rightarrow}\right)$ is continuous on (L_2 $\left.(\partial \Psi)\right)^{\wedge} 3$ for each $\mathrm{P}=0,1,2$.Proof: For any $\mathrm{P}=0,1,2$, we set

$\mathrm{P}_{\mathrm{P}_{1}}(\mathrm{x}, \overrightarrow{\mathrm{b}})=\mathrm{G}_{\mathrm{P} 1}\left(\mathrm{x}, \mathrm{b}_{1}\right)+\mathrm{G}_{\mathrm{P} 2}\left(\mathrm{x}, \mathrm{b}_{2}\right)+\mathrm{G}_{\mathrm{P} 3}\left(\mathrm{x}, \mathrm{b}_{3}\right)$ and

$\mathrm{p}_{\mathrm{P}_{2}}(\mathrm{x}, \overrightarrow{\mathrm{d}})=\mathrm{G}_{\mathrm{P} 4}\left(\mathrm{x}, \mathrm{d}_{1}\right)+\mathrm{G}_{\mathrm{P} 5}\left(\mathrm{x}, \mathrm{d}_{2}\right)+\mathrm{G}_{\mathrm{P} 6}\left(\mathrm{x}, \mathrm{d}_{3}\right)$.

To prove the continuity for any one of the above two integrals, the used technique will be similar. Thus, it is enough to prove one of them, which is in this case the second integral. Hence, let $\vec{d}=$ $\left(d_{1}, d_{2}, d_{3}\right)$, with $p_{P_{2}}: \Psi \times \mathbb{R}^{3} \rightarrow \mathbb{R}$, then from assumption (II), we have

$$
\begin{aligned}
\left\|p_{\mathrm{P}_{2}}(\mathrm{x}, \overrightarrow{\mathrm{d}})\right\| & \leq \Upsilon_{\mathrm{P}_{7}}(\mathrm{x})+c_{\mathrm{P} 4} \mathrm{~d}_{1}^{2}+c_{\mathrm{P} 5} \mathrm{~d}_{2}^{2}+c_{\mathrm{P} 6} \mathrm{~d}_{3}^{2} \\
& \leq \Upsilon_{\mathrm{P}_{7}}(\mathrm{x})+c_{\mathrm{P} 7}\|\overrightarrow{\mathrm{d}}\|^{2}
\end{aligned}
$$

where $\Upsilon_{\mathrm{P}_{7}}=\Upsilon_{\mathrm{P}_{4}}+\Upsilon_{\mathrm{P}_{5}}+\Upsilon_{\mathrm{P}_{6}}, c_{\mathrm{P} 7}=\max \left(c_{\mathrm{P} 4}, c_{\mathrm{P} 5}, c_{\mathrm{P} 6}\right), \Upsilon_{\mathrm{P}_{7}} \in \mathrm{L}_{1}(\partial \Psi), c_{\mathrm{P} 7} \in \mathrm{L}_{\infty}(\mathbb{R})$.

Then, the $\int_{\partial \Psi} \mathrm{P}_{\mathrm{P}_{2}}(\mathrm{x}, \overrightarrow{\mathrm{d}}) \mathrm{d} \gamma$ is continuous on $\left(\mathrm{L}_{2}(\partial \Psi)\right)^{3}$ (by using Proposition (1) in reference [19]). Hence,

$\mathrm{T}_{\mathrm{P}}(\overrightarrow{\mathrm{d}})=\iint_{\Psi} \mathrm{p}_{\mathrm{P}_{1}}(\mathrm{x}, \overrightarrow{\mathrm{b}}) \mathrm{dx}_{1} \mathrm{dx}_{2}+\int_{\partial \Psi} \mathrm{p}_{\mathrm{P}_{2}}(\mathrm{x}, \overrightarrow{\mathrm{d}}) \mathrm{d} \gamma$ is continuous on $\left(\mathrm{L}_{2}(\partial \Psi)\right)^{3}$.

Theorem (2): If the assumptions (I) and (II) are hold, $\overrightarrow{\mathrm{E}}_{\mathrm{A}} \neq \varnothing, \mathrm{m}_{1}, \mathrm{~m}_{2}, \mathrm{~m}_{3}$ are not dependent on $\mathrm{d}_{1}, \mathrm{~d}_{2}, \mathrm{~d}_{3}$, respectively, and $\mathrm{5}_{1}, \mathrm{~K}_{2}, \mathrm{~K}_{3}$ are bounded functions, so that,

$\left|\mathrm{m}_{1}\left(\mathrm{x}, \mathrm{b}_{1}\right)\right| \leq \emptyset_{1(\mathrm{x})}+\overline{\mathrm{c}}_{1}\left|\mathrm{~b}_{1}\right|,\left|\mathrm{m}_{2}\left(\mathrm{x}, \mathrm{b}_{2}\right)\right| \leq \emptyset_{2(\mathrm{x})}+\bar{c}_{2}\left|\mathrm{~b}_{2}\right|,\left|\mathrm{m}_{3}\left(\mathrm{x}, \mathrm{b}_{3}\right)\right| \leq \emptyset_{3(\mathrm{x})}+\overline{\mathrm{c}}_{3}\left|\mathrm{~b}_{3}\right|$,

$\left|\varsigma_{1}(\mathrm{x})\right| \leq \mathrm{m}_{1},\left|\varsigma_{2}(\mathrm{x})\right| \leq \mathrm{m}_{2}$, and $\left|\mathrm{\zeta}_{3}(\mathrm{x})\right| \leq \mathrm{m}_{3}$,

where $\emptyset_{\sigma} \in \mathrm{L}_{2}(\Psi), \overline{\mathrm{c}}_{\sigma} \geq 0$, and $\mathrm{m}_{\mathrm{i}} \geq 0$, for $\sigma=1,2,3$.

$\mathrm{J}_{11}, \mathrm{G}_{12}, \mathrm{t}_{13}$ are not dependent on $\mathrm{d}_{1}, \mathrm{~d}_{2} \mathrm{~d}_{3}$, respectively. $\mathrm{G}_{\mathrm{P} 4}, \mathrm{~T}_{\mathrm{P} 5}, \mathrm{~T}_{\mathrm{P} 6}(\mathrm{P}=0,2)$ are convex with respect to $d_{1}, d_{2}, d_{3}$, respectively, for fixed $x$. Then there exists a continuous classical boundary optimal control vector.

Proof: The set E_ $\sigma$ and $\mathrm{D} \_\sigma(\forall \sigma=1,2,3)$ is convex and bounded, then E_1 $1 \times \mathrm{E} \_2 \times \mathrm{E} \_3$ is convex and bounded. On the other hand, by using theorem (2) in reference [19], E_ $\sigma \forall \sigma=1,2,3$ is closed, since D_ $\sigma$ is closed, then E_1 $1 \times \mathrm{E}_{-} 2 \times \mathrm{E} \_3$ is closed, too. Therefore, we obtain that E_1 $1 \times \mathrm{E}_{-} 2 \times \mathrm{E} \_3$ is weakly compact.

From the assumption on $\vec{E}_{A}$, there is an element $\vec{e} \in \vec{E}_{A}$. Then there is a minimum sequence $\left\{\vec{d}_{n}\right\}=$ $\left\{\left(\mathrm{d}_{1 \mathrm{n}}, \mathrm{d}_{2 \mathrm{n}}, \mathrm{d}_{3 \mathrm{n}}\right)\right\} \in \overrightarrow{\mathrm{E}}_{\mathrm{A}}$ for each $\mathrm{n}$, with $\mathrm{G}_{1}\left(\overrightarrow{\mathrm{d}}_{\mathrm{n}}\right)=0, \mathrm{~T}_{2}\left(\overrightarrow{\mathrm{d}}_{\mathrm{n}}\right) \leq 0$, , so that

$\lim _{\mathrm{n} \rightarrow \infty} \mathrm{T}_{0}\left(\overrightarrow{\mathrm{d}}_{\mathrm{n}}\right)=\inf _{\overrightarrow{\mathrm{e}} \in \overrightarrow{\mathrm{E}}} \mathrm{T}_{0}(\overrightarrow{\mathrm{e}})$.

But $\vec{E}$ is weakly compact, then there is a subsequence of $\left\{\vec{d}_{n}\right\}$, which will be symbolized again by $\left\{\overrightarrow{\mathrm{d}}_{\mathrm{n}}\right\}$, that converges weakly to $\overrightarrow{\mathrm{d}}$ in $\overrightarrow{\mathrm{E}}$.

Then, corresponding to the $\left\{\vec{d}_{n}\right\}$, there is the sequence of the "state" solution vector $\left\{\vec{b}_{n}\right\}$ of the sequence of the weak form. Then, from the proof of Theorem (3), we have:

$$
\begin{aligned}
& \mathrm{a}_{1}\left(\mathrm{~b}_{1 \mathrm{n}}, \mathrm{t}_{1}\right)-\left(\mathrm{b}_{2 \mathrm{n}}+\mathrm{b}_{3 \mathrm{n}}, \mathrm{t}_{1}\right)_{\mathrm{L}_{2}(\Psi)}+\mathrm{a}_{2}\left(\mathrm{~b}_{2 \mathrm{n}}, \mathrm{t}_{2}\right)+\left(\mathrm{b}_{1 \mathrm{n}}+\mathrm{b}_{3 \mathrm{n}}, \mathrm{t}_{2}\right)_{\mathrm{L}_{2}(\Psi)} \\
& \left(\mathrm{b}_{1 \mathrm{n}}-\mathrm{b}_{2 \mathrm{n}}, \mathrm{t}_{3}\right)_{\mathrm{L}_{2}(\Psi)}+\left(\mathrm{m}_{1}\left(\mathrm{x}, \mathrm{b}_{1 \mathrm{n}}\right), \mathrm{t}_{1}\right)_{\mathrm{L}_{2}(\Psi)}+\left(\mathrm{m}_{2}\left(\mathrm{x}, \mathrm{b}_{2 \mathrm{n}}\right), \mathrm{t}_{2}\right)_{\mathrm{L}_{2}(\Psi)} \\
& +\left(\mathrm{m}_{3}\left(\mathrm{x}, \mathrm{b}_{3 \mathrm{n}}\right), \mathrm{t}_{3}\right)_{\mathrm{L}_{2}(\Psi)} \\
& =\left(\mathrm{r}_{1}(\mathrm{x}), \mathrm{t}_{1}\right)_{\mathrm{L}_{2}(\Psi)}+\left(\mathrm{d}_{1 \mathrm{n}}, \mathrm{t}_{3}\right)_{\mathrm{L}_{2}(\partial \Psi)}+\left(\mathrm{s}_{2}(\mathrm{x}), \mathrm{t}_{2}\right)_{\mathrm{L}_{2}(\Psi)}+\left(\mathrm{d}_{2 \mathrm{n}}, \mathrm{t}_{2}\right)_{\mathrm{L}_{2}(\partial \Psi)} \\
& \quad+\left(\mathrm{s}_{3}(\mathrm{x}), \mathrm{t}_{3}\right)_{\mathrm{L}_{2}(\Psi)}+\left(\mathrm{d}_{3 \mathrm{n}}, \mathrm{t}_{3}\right)_{\mathrm{L}_{2}(\partial \Psi)}
\end{aligned}
$$

With $\left\|\vec{b}_{n}\right\|_{\left(H^{1}(\Psi)\right)^{3}}$ for each $n$ is bounded, then $\left\{\vec{b}_{n}\right\}$ has a subsequence, which will be symbolized again by $\left\{\vec{b}_{n}\right\}$, such that $\vec{b}_{n} \rightarrow \vec{b}$ weakly in $\vec{V}$ (Alaoglu theorem [20]).

Now, we have to show that (20) converges to

$$
\begin{aligned}
& \mathrm{a}_{1}\left(\mathrm{~b}_{1}, \mathrm{t}_{1}\right)-\left(\mathrm{b}_{2}+\mathrm{b}_{3}, \mathrm{t}_{1}\right)_{\mathrm{L}_{2}(\Psi)}+\mathrm{a}_{2}\left(\mathrm{~b}_{2}, \mathrm{t}_{2}\right)+\left(\mathrm{b}_{1}+\mathrm{b}_{3}, \mathrm{t}_{2}\right)_{\mathrm{L}_{2}(\Psi)}+\mathrm{a}_{3}\left(\mathrm{~b}_{3}, \mathrm{t}_{3}\right) \\
& \left.\mathrm{b}_{2}, \mathrm{t}_{3}\right)_{\mathrm{L}_{2}(\Psi)}+\left(\mathrm{m}_{1}\left(\mathrm{x}, \mathrm{b}_{1}\right), \mathrm{t}_{1}\right)_{\mathrm{L}_{2}(\Psi)}+\left(\mathrm{m}_{2}\left(\mathrm{x}, \mathrm{b}_{2}\right), \mathrm{t}_{2}\right)_{\mathrm{L}_{2}(\Psi)}+\left(\mathrm{m}_{3}\left(\mathrm{x}, \mathrm{b}_{3}\right), \mathrm{t}_{3}\right)_{\mathrm{L}_{2}(\Psi)} \\
& =\left(\mathrm{s}_{1}(\mathrm{x}), \mathrm{t}_{1}\right)_{\mathrm{L}_{2}(\Psi)}+\left(\mathrm{d}_{1}, \mathrm{t}_{3}\right)_{\mathrm{L}_{2}(\partial \Psi)}+\left(\mathrm{b}_{2}(\mathrm{x}), \mathrm{t}_{2}\right)_{\mathrm{L}_{2}(\Psi)}+\left(\mathrm{d}_{2}, \mathrm{t}_{2}\right)_{\mathrm{L}_{2}(\partial \Psi)}+\left(\mathrm{s}_{3}(\mathrm{x}), \mathrm{t}_{3}\right)_{\mathrm{L}_{2}(\Psi)} \\
& \quad+\left(\mathrm{d}_{3}, \mathrm{t}_{3}\right)_{\mathrm{L}_{2}(\partial \Psi)}
\end{aligned}
$$


First, let $\left(t_{1}, t_{2}, t_{3}\right) \in(C(\bar{\Psi}))^{3}$, and, first for the left hand side, since $b_{\sigma n} \rightarrow b_{\sigma}$ weakly in $T_{\sigma}$, i.e $\mathrm{b}_{\sigma \mathrm{n}} \rightarrow \mathrm{b}_{\sigma}$ weakly in $\mathrm{L}_{2}(\Psi)$, for each $\sigma=1,2,3$, then from the left hand side of (20) and

(21) and by using the Cauchy-Schwarz inequality, one has

$$
\begin{aligned}
& \mid a_{1}\left(b_{1 n}, t_{1}\right)-\left(b_{2 n}+b_{3 n}, t_{1}\right)_{L_{2}(\Psi)}+a_{2}\left(b_{2 n}, t_{2}\right)+\left(b_{1 n}+b_{3 n}, t_{2}\right)_{L_{2}(\Psi)} \\
& +a_{3}\left(b_{3 n}, t_{3}\right)+\left(b_{1 n}-b_{2 n}, t_{3}\right)_{L_{2}(\Psi)}-a_{1}\left(b_{1}, t_{1}\right)+\left(b_{2}+b_{3}, t_{1}\right)_{L_{2}(\Psi)} \\
& -a_{2}\left(b_{2}, t_{2}\right)-\left(b_{1}+b_{3}, t_{2}\right)_{L_{2}(\Psi)}-a_{3}\left(b_{3}, t_{3}\right)-\left(b_{1}-b_{2}, t_{3}\right)_{L_{2}(\Psi)} \mid \\
& \quad \leq\left(c_{1}\left\|b_{1 n}-b_{1}\right\|_{H^{1}(\Psi)}+\left\|b_{2 n}-b_{2}\right\|_{L_{2}(\Psi)}+\left\|b_{3 n}-b_{3}\right\|_{L_{2}(\Psi)}\right)\left\|t_{1}\right\|_{L_{2}(\Psi)}+ \\
& +\left(c_{2}\left\|b_{2 n}-b_{2}\right\|_{H^{1}(\Psi)}+\left\|b_{1 n}-b_{1}\right\|_{L_{2}(\Psi)}+\left\|b_{3 n}-b_{3}\right\|_{L_{2}(\Psi)}\right)\left\|t_{2}\right\|_{L_{2}(\Psi)}+ \\
& \left(c_{3}\left\|b_{3 n}-b_{3}\right\|_{H^{1}(\Psi)}+\left\|b_{1 n}-b_{1}\right\|_{L_{2}(\Psi)}+\left\|b_{2 n}-b_{2}\right\|_{L_{2}(\Psi)}\right)\left\|t_{3}\right\|_{L_{2}(\Psi)} \rightarrow 0
\end{aligned}
$$

i. From assumption (II) and Proposition (1), the functions $\iint_{\Psi} \mathrm{m}_{1}\left(\mathrm{x}, \mathrm{b}_{1 \mathrm{n}}\right) \mathrm{t}_{1} \mathrm{~d} \mathrm{x}_{1} \mathrm{~d} \mathrm{x}_{2}$ $\iint_{\Psi} m_{2}\left(x, b_{2 n}\right) t_{2} d x_{1} d x_{2}$ and $\iint_{\Psi} m_{3}\left(x, b_{3 n}\right) t_{3} d x_{1} d x_{2}$ are continuous with respect to $b_{1 n}, b_{2 n}$ and $\mathrm{b}_{3 \mathrm{n}}$, respectively.

But $\vec{b}_{n} \rightarrow \vec{b}$ weakly in $\left(L_{2}(\Psi)\right)^{3}$, because $\vec{b}_{n} \rightarrow \vec{b}$ weakly in $\vec{T}$, then by using the RellichKondrachov theorem in [21], we get that $\vec{b}_{n} \rightarrow \vec{b}$ strongly in $\left(L_{2}(\Psi)\right)^{3}$, hence

$$
\begin{aligned}
\left(\mathrm{m}_{1}\left(\mathrm{x}, \mathrm{b}_{1 \mathrm{n}}\right), \mathrm{t}_{1}\right)_{\mathrm{L}_{2}(\Psi)}+\left(\mathrm{m}_{2}\left(\mathrm{x}, \mathrm{b}_{2 \mathrm{n}}\right), \mathrm{t}_{2}\right)_{\mathrm{L}_{2}(\Psi)}+\left(\mathrm{m}_{3}\left(\mathrm{x}, \mathrm{b}_{3 \mathrm{n}}\right), \mathrm{t}_{3}\right)_{\mathrm{L}_{2}(\Psi)} & \\
& \rightarrow\left(\mathrm{m}_{1}\left(\mathrm{x}, \mathrm{b}_{1}\right), \mathrm{t}_{1}\right)_{\mathrm{L}_{2}(\Psi)}+\left(\mathrm{m}_{2}\left(\mathrm{x}, \mathrm{b}_{2}\right), \mathrm{t}_{2}\right)_{\mathrm{L}_{2}(\Psi)}+\left(\mathrm{m}_{3}\left(\mathrm{x}, \mathrm{b}_{3}\right), \mathrm{t}_{3}\right)_{\mathrm{L}_{2}(\Psi)}
\end{aligned}
$$

i.e. the left hand side of $(20) \rightarrow$ the left hand side of (21).

Second, since $d_{1 n} \rightarrow d_{1}, d_{2 n} \rightarrow d_{2}$ and $d_{3 n} \rightarrow d_{3}$ weakly in $L_{2}(\partial \Psi)$, then

$$
\left(\mathrm{d}_{1 \mathrm{n}}-\mathrm{d}_{1}, \mathrm{t}_{1}\right)_{\mathrm{L}_{2}(\partial \Psi)}+\left(\mathrm{d}_{2 \mathrm{n}}-\mathrm{d}_{2}, \mathrm{t}_{2}\right)_{\mathrm{L}_{2}(\partial \Psi)}+\left(\mathrm{d}_{3 \mathrm{n}}-\mathrm{d}_{3}, \mathrm{t}_{3}\right)_{\mathrm{L}_{2}(\partial \Psi)} \rightarrow 0
$$

From (23a) and (23b), we obtain that (20) converges to (21).

Since $(C(\bar{\Psi}))^{3}$ is dense in $\vec{V}$, then this convergence satisfies for any $\left(t_{1}, t_{2}, t_{3}\right) \in \vec{T}$. This leads to $\vec{b}_{\mathrm{n}} \rightarrow \overrightarrow{\mathrm{b}}=\overrightarrow{\mathrm{b}}_{\overrightarrow{\mathrm{d}}}$ is a solution of the weak form of the triple state equations.

From Lemma (2), the functional $\mathrm{G}_{\mathrm{P}}(\overrightarrow{\mathrm{d}})$ is continuous on $\left(\mathrm{L}_{2}(\partial \Psi)\right)^{3}, \forall \mathrm{P}=0,1,2$.

From the assumptions on $\mathrm{v}_{11}, \mathrm{G}_{12}, \mathrm{t}_{13}, \mathrm{G}_{1}\left(\overrightarrow{\mathrm{d}}_{\mathrm{n}}\right)$ is continuous and from the strongly converged

$\mathrm{b}_{1 \mathrm{n}} \rightarrow \mathrm{b}_{1}, \mathrm{~b}_{2 \mathrm{n}} \rightarrow \mathrm{b}_{2}$ and $\mathrm{b}_{3 \mathrm{n}} \rightarrow \mathrm{b}_{3}$ in $\mathrm{L}_{2}(\Psi)$, we get

$\mathrm{T}_{1}(\overrightarrow{\mathrm{d}})=\lim _{\mathrm{n} \rightarrow \infty} \mathrm{T}_{1}\left(\overrightarrow{\mathrm{d}}_{\mathrm{n}}\right)=0$.

Also, from the assumptions on $\mathrm{G}_{\mathrm{P} 1}\left(\mathrm{x}, \mathrm{b}_{1}\right)$ and $\mathrm{T}_{\mathrm{P} 4}\left(\mathrm{x}, \mathrm{d}_{1}\right)(\forall \mathrm{P}=0,2)$ and Lemma (2), the integrals $\iint_{\Psi^{\top} \mathrm{G}_{P 1}}\left(\mathrm{x}, \mathrm{b}_{1}\right) \mathrm{dx}_{1} \mathrm{dx}_{2}$ and $\int_{\partial \Psi \mathrm{G}_{\mathrm{P}}}\left(\mathrm{x}, \mathrm{d}_{1}\right) \mathrm{d} \gamma$ are continuous with respect to $\mathrm{b}_{1}$ and $\mathrm{d}_{1}$, respectively, but $\mathrm{T}_{\mathrm{P}_{4}}\left(\mathrm{x}, \mathrm{d}_{1}\right),(\forall \mathrm{P}=0,2)$ is convex with respect to $\mathrm{d}_{1}$, then $\int_{\partial \Psi^{\top} \mathrm{T}_{\mathrm{P} 4}}\left(\mathrm{x}, \mathrm{d}_{1}\right) \mathrm{d} \gamma$ is weakly lower semicontinuous with respect to $d_{1}$, i.e.

$\iint_{\Psi} \mathrm{G}_{\mathrm{P} 1}\left(\mathrm{x}, \mathrm{b}_{1}\right) \mathrm{dx}_{1} \mathrm{dx}_{2}+\int_{\partial \Psi} \mathrm{G}_{\mathrm{P} 4}\left(\mathrm{x}, \mathrm{d}_{1}\right) \mathrm{d} \gamma$

$\leq \iint_{\Psi} \mathbf{G}_{\mathrm{P} 1}\left(\mathrm{x}, \mathrm{b}_{1}\right) \mathrm{dx}_{1} \mathrm{dx}_{2}+\frac{\lim }{\mathrm{n} \rightarrow \infty} \int_{\partial \Psi} \mathbf{G}_{\mathrm{P} 4}\left(\mathrm{x}, \mathrm{d}_{1 \mathrm{n}}\right) \mathrm{d} \gamma$

$=\frac{\lim }{n \rightarrow \infty} \iint_{\Psi}\left[\mathrm{G}_{\mathrm{P} 1}\left(\mathrm{x}, \mathrm{b}_{1}\right)-\mathrm{T}_{\mathrm{P} 1}\left(\mathrm{x}, \mathrm{b}_{1 \mathrm{n}}\right)\right] \mathrm{d} \mathrm{x}_{1} \mathrm{~d} \mathrm{x}_{2}$

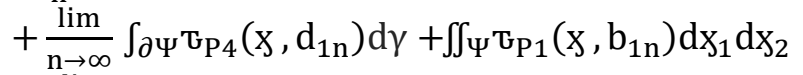

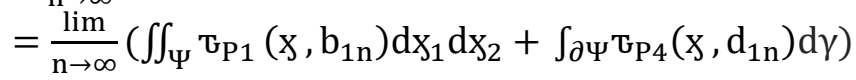

By the same manner, and for each $\mathrm{P}=0,2$, we get the following two convergences:

$$
\begin{aligned}
& \iint_{\Psi} \mathrm{G}_{\mathrm{P} 2}\left(\mathrm{x}, \mathrm{b}_{2}\right) \mathrm{dx}_{1} \mathrm{dx}_{2}+\int_{\partial \Psi} \mathrm{G}_{\mathrm{P} 5}\left(\mathrm{x}, \mathrm{d}_{2}\right) \mathrm{d} \gamma \\
& \leq \frac{\lim }{\mathrm{n} \rightarrow \infty}\left(\iint_{\Psi} \mathrm{G}_{\mathrm{P} 2}\left(\mathrm{x}, \mathrm{b}_{2 \mathrm{n}}\right) \mathrm{dx}_{1} \mathrm{dx}_{2}+\int_{\partial \Psi} \mathrm{G}_{\mathrm{P} 5}\left(\mathrm{x}, \mathrm{d}_{2 \mathrm{n}}\right) \mathrm{d} \gamma\right) \\
& \text { and } \\
& \iint_{\Psi} \mathrm{T}_{\mathrm{P} 3}\left(\mathrm{x}, \mathrm{b}_{3}\right) \mathrm{dx}_{1} \mathrm{dx}_{2}+\int_{\partial \Psi} \mathrm{G}_{\mathrm{P} 6}\left(\mathrm{x}, \mathrm{d}_{3}\right) \mathrm{d} \gamma \\
& \leq \frac{\lim }{\mathrm{n} \rightarrow \infty}\left(\iint_{\Psi} \mathrm{G}_{\mathrm{P} 3}\left(\mathrm{x}, \mathrm{b}_{3 \mathrm{n}}\right) \mathrm{dx}_{1} \mathrm{dx}_{2}+\int_{\partial \Psi} \mathbf{G}_{\mathrm{P} 6}\left(\mathrm{x}, \mathrm{d}_{3 \mathrm{n}}\right) \mathrm{d} \gamma\right)
\end{aligned}
$$

From the above inequalities, one gets that $\mathrm{G}_{\mathrm{P} \ell}(\overrightarrow{\mathrm{d}}),(\forall \mathrm{P}=0,2)$ is weakly lower semicontinuous with respect to $(\vec{b}, \vec{d})$. Thus $\mathrm{T}_{2}(\overrightarrow{\mathrm{d}}) \leq \frac{\lim }{\mathrm{n} \rightarrow \infty} \mathrm{T}_{2}\left(\overrightarrow{\mathrm{d}}_{\mathrm{n}}\right) \leq 0$, and 
$\mathrm{T}_{0}(\overrightarrow{\mathrm{d}}) \leq \frac{\lim }{\mathrm{n} \rightarrow \infty} \mathrm{T}_{0}\left(\overrightarrow{\mathrm{d}}_{\mathrm{n}}\right)=\lim _{\mathrm{n} \rightarrow \infty} \mathrm{T}_{0}\left(\overrightarrow{\mathrm{d}}_{\mathrm{n}}\right)=\inf _{\overrightarrow{\mathrm{w}} \in \overrightarrow{\mathrm{w}}} \mathrm{T}_{0}(\overrightarrow{\mathrm{w}}) \Rightarrow$

$\overrightarrow{\mathrm{d}}$ is a continuous classical boundary optimal control vector .

\section{The Necessary and Sufficient Conditions for Optimality of the Continuous Classical Boundary Optimal Control Vector}

The following assumptions are useful in this section to derive the Fréchet derivative of the Hamiltonian.

\section{Assumption (III)}

a) $\mathrm{m}_{1_{\mathrm{b}_{1}}}, \mathrm{~m}_{2 \mathrm{~b}_{2}}, \mathrm{~m}_{3_{\mathrm{b}_{3}}}$ are of the Carathéodory type on $\Psi \times \mathbb{R}$ and satisfy

$$
\begin{aligned}
& \left|\mathrm{m}_{1 \mathrm{~b}_{1}}\left(\mathrm{x}, \mathrm{b}_{1}\right)\right| \leq \breve{c}_{1},\left|\mathrm{~m}_{2 \mathrm{~b}_{2}}\left(\mathrm{x}, \mathrm{b}_{2}\right)\right| \leq \breve{c}_{2},\left|\mathrm{~m}_{3 \mathrm{~b}_{3}}\left(\mathrm{x}, \mathrm{b}_{3}\right)\right| \leq \breve{c}_{3}, \text { with } \breve{c}_{1}, \breve{c}_{2}, \breve{c}_{3} \geq 0 \\
& \mathrm{~m}_{1_{\mathrm{b}_{1}}}\left(\mathrm{x}, \mathrm{b}_{1}\right) \geq 0, \mathrm{~m}_{2 \mathrm{~b}_{2}}\left(\mathrm{x}, \mathrm{b}_{2}\right) \geq 0 \text { and } \mathrm{m}_{3_{\mathrm{b}_{3}}}\left(\mathrm{x}, \mathrm{b}_{3}\right) \geq 0 .
\end{aligned}
$$

b) $5_{1}, 5_{2}, 5_{3}$ are of the Carathéodory type on $\Psi$ and satisfy

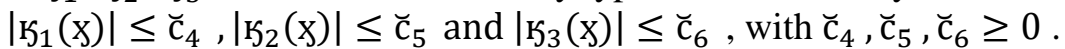

c) $\mathrm{T}_{\mathrm{P}_{1} \mathrm{~b}_{1}}, \mathrm{~T}_{\mathrm{P}_{2} \mathrm{~b}_{2}}, \mathrm{~T}_{\mathrm{P}_{3} \mathrm{~b}_{3}}, \mathrm{~T}_{\mathrm{P}_{4} \mathrm{~d}_{1}}, \mathrm{~T}_{\mathrm{P}_{\mathrm{d}_{2}}}, \mathrm{~T}_{\mathrm{P} 6_{\mathrm{d}_{3}}}(\forall \mathrm{P}=0,1,2)$ are of the Carathéodory type on $\Psi \times \mathbb{R}$ and satisfy

$$
\begin{aligned}
& \left|{ }^{\mathrm{G}_{\mathrm{P}_{1} \mathrm{~b}_{1}}}\right| \leq \Upsilon_{\mathrm{P}_{1}}+c_{\mathrm{P} 1}\left|\mathrm{~b}_{1}\right|,\left|\mathrm{G}_{\mathrm{P}_{2} \mathrm{~b}_{2}}\right| \leq \Upsilon_{\mathrm{P}_{2}}+c_{\mathrm{P} 2}\left|\mathrm{~b}_{1}\right|,\left|\mathrm{G}_{\mathrm{P}_{3} \mathrm{~b}_{3}}\right| \leq r_{\ell_{3}}+c_{\mathrm{P} 3}\left|\mathrm{~b}_{1}\right|, \\
& \left|\mathrm{G}_{\mathrm{P}_{4} \mathrm{~d}_{1}}\right| \leq \Upsilon_{\mathrm{P}_{4}}+c_{\mathrm{P} 4}\left|\mathrm{~d}_{1}\right|,\left|\mathrm{G}_{\mathrm{P}_{5} \mathrm{~d}_{2}}\right| \leq r_{\mathrm{P}_{5}}+c_{\mathrm{P} 5}\left|\mathrm{~d}_{2}\right| \text {, and }\left|\mathrm{G}_{\mathrm{P}_{6} \mathrm{~d}_{3}}\right| \leq \Upsilon_{\mathrm{P}_{6}}+c_{\mathrm{P}_{6}}\left|\mathrm{~d}_{3}\right|
\end{aligned}
$$

where $c_{\mathrm{P} \sigma} \geq 0, \Upsilon_{\mathrm{P}_{1}}, \Upsilon_{\mathrm{P}_{2}}, \Upsilon_{\mathrm{P}_{3}} \in \mathrm{L}_{2}(\Psi)$ and $\Upsilon_{\mathrm{P}_{4}}, \Upsilon_{\mathrm{P}_{5}}, \Upsilon_{\mathrm{P}_{6}} \in \mathrm{L}_{2}(\partial \Psi)$, for $\sigma=1,2,3,4,5,6$ and $\mathrm{P}=$ $0,1,2$.

Theorem (3): If the assumptions (I), (II), and (III) are hold, the Hamiltonian is given as:

$$
\begin{aligned}
& \text { U }\left(\mathrm{x}, \mathrm{b}_{1}, \mathrm{~b}_{2}, \mathrm{~b}_{3}, \mathrm{z}_{1}, \mathrm{z}_{2}, \mathrm{z}_{3}, \mathrm{~d}_{1}, \mathrm{~d}_{2}, \mathrm{~d}_{3}\right) \\
& =\mathrm{z}_{1}\left(\mathrm{~s}_{1}(\mathrm{x})-\mathrm{m}_{1}\left(\mathrm{x}, \mathrm{b}_{1}\right)\right)+\mathrm{w}_{01}\left(\mathrm{x}, \mathrm{b}_{1}\right)+\mathrm{t}_{04}\left(\mathrm{x}, \mathrm{d}_{1}\right)+\mathrm{z}_{2}\left(\mathrm{~s}_{2}(\mathrm{x})-\mathrm{m}_{2}\left(\mathrm{x}, \mathrm{b}_{2}\right)\right) \\
& \quad+\mathrm{w}_{02}\left(\mathrm{x}, \mathrm{b}_{2}\right)+\mathrm{t}_{05}\left(\mathrm{x}, \mathrm{d}_{2}\right)+\mathrm{z}_{3}\left(\mathrm{~s}_{3}(\mathrm{x})-\mathrm{m}_{3}\left(\mathrm{x}, \mathrm{b}_{3}\right)\right)+\mathrm{w}_{03}\left(\mathrm{x}, \mathrm{b}_{3}\right)+\mathrm{w}_{06}\left(\mathrm{x}, \mathrm{d}_{3}\right)
\end{aligned}
$$

The triple adjoint equations of the triple state equations (1-6) are :

$$
\begin{aligned}
& \mathrm{A}_{1} \mathrm{z}_{1}+\mathrm{z}_{1}+\mathrm{z}_{2}+\mathrm{z}_{3}+\mathrm{z}_{1} \mathrm{~m}_{1 \mathrm{~b}_{1}}\left(\mathrm{x}, \mathrm{b}_{1}\right)=\mathrm{w}_{01 \mathrm{~b}_{1}}\left(\mathrm{x}, \mathrm{b}_{1}\right), \text { in } \Psi \\
& \mathrm{A}_{2} \mathrm{z}_{2}-\mathrm{z}_{1}+\mathrm{z}_{2}-\mathrm{z}_{3}+\mathrm{z}_{2} \mathrm{~m}_{2 \mathrm{~b}_{2}}\left(\mathrm{x}, \mathrm{b}_{2}\right)=\mathrm{t}_{02 \mathrm{~b}_{2}}\left(\mathrm{x}, \mathrm{b}_{2}\right), \text { in } \Psi \\
& \mathrm{A}_{3} \mathrm{z}_{3}-\mathrm{z}_{1}+\mathrm{z}_{2}+\mathrm{z}_{3}+\mathrm{z}_{3} \mathrm{~m}_{3 \mathrm{~b}_{3}}\left(\mathrm{x}, \mathrm{b}_{3}\right)=\mathrm{t}_{03 \mathrm{~b}_{3}}\left(\mathrm{x}, \mathrm{b}_{3}\right), \text { in } \Psi \\
& \frac{\partial \mathrm{z}_{1}}{\partial \mathrm{n}_{1}}=0 \text {, in } \partial \Psi \\
& \frac{\partial \mathrm{z}_{2}}{\partial \mathrm{n}_{2}}=0 \text {, in } \partial \Psi \\
& \frac{\partial \mathrm{z}_{3}}{\partial \mathrm{n}_{3}}=0 \text {, in } \partial \Psi
\end{aligned}
$$

Then the Fréchet derivative of $\mathrm{T}_{0}$ is

$\overrightarrow{\mathrm{G}_{0}^{\prime}}(\overrightarrow{\mathrm{d}}) \cdot \overrightarrow{\Delta \mathrm{d}}=\int_{\partial \Psi} \mathrm{h}_{\overrightarrow{\mathrm{d}}}^{\mathrm{T}} \cdot \overrightarrow{\Delta \mathrm{d}} \mathrm{d} \gamma$, where

$U_{\vec{d}}^{T}=\left(\begin{array}{l}U_{d_{1}}\left(x, z_{1}, z_{2}, z_{3}, d_{1}, d_{2}, d_{3}\right) \\ U_{d_{2}}\left(x, z_{1}, z_{2}, z_{3}, d_{1}, d_{2}, d_{3}\right) \\ U_{d_{3}}\left(x, z_{1}, z_{2}, z_{3}, d_{1}, d_{2}, d_{3}\right)\end{array}\right)=\left(\begin{array}{l}z_{1}+\tau_{04 d_{1}} \\ z_{2}+\tau_{05} d_{2} \\ z_{3}+\tau_{06 d_{3}}\end{array}\right)$ and $\vec{z}=\vec{z}_{\vec{d}}$ is the triple adjoint equation of the triple state equation $\vec{y}_{\vec{d}}$.

Proof: Formulating the triple adjoint equations (24-29) by their weak forms, then adding them, and then setting $\vec{t}=\overrightarrow{\Delta b}$ in the resulting equation, yield

$$
\begin{aligned}
& \mathrm{a}_{1}\left(\mathrm{z}_{1}, \Delta \mathrm{b}_{1}\right)+\left(\mathrm{z}_{2}+\mathrm{z}_{3}, \Delta \mathrm{b}_{1}\right)_{\mathrm{L}_{2}(\Psi)}+\mathrm{a}_{2}\left(\mathrm{z}_{2}, \Delta \mathrm{b}_{2}\right)-\left(\mathrm{z}_{1}+\mathrm{z}_{3}, \Delta \mathrm{b}_{2}\right)_{\mathrm{L}_{2}(\Psi)}+\mathrm{a}_{3}\left(\mathrm{z}_{3}, \Delta \mathrm{b}_{3}\right) \\
& -\left(\mathrm{z}_{1}-\mathrm{z}_{2}, \Delta \mathrm{b}_{3}\right)_{\mathrm{L}_{2}(\Psi)}+\left(\mathrm{z}_{1} \mathrm{~m}_{1 \mathrm{~b}_{1}}\left(\mathrm{~b}_{1}\right), \Delta \mathrm{b}_{1}\right)_{\mathrm{L}_{2}(\Psi)}+\left(\mathrm{z}_{2} \mathrm{~m}_{2 \mathrm{~b}_{2}}\left(\mathrm{~b}_{2}\right), \Delta \mathrm{b}_{2}\right)_{\mathrm{L}_{2}(\Psi)} \\
& +\left(\mathrm{z}_{3} \mathrm{~m}_{3 \mathrm{~b}_{3}}\left(\mathrm{~b}_{3}\right), \Delta \mathrm{b}_{3}\right)_{\mathrm{L}_{2}(\Psi)}=\left(\mathrm{v}_{01 \mathrm{~b}_{1}}\left(\mathrm{~b}_{1}\right), \Delta \mathrm{b}_{1}\right)_{\mathrm{L}_{2}(\Psi)} \\
& +\left(\mathrm{G}_{02 \mathrm{~b}_{2}}\left(\mathrm{~b}_{2}\right), \Delta \mathrm{b}_{2}\right)_{\mathrm{L}_{2}(\Psi)}+\left(\mathrm{T}_{03 \mathrm{~b}_{3}}\left(\mathrm{~b}_{3}\right), \Delta \mathrm{b}_{3}\right)_{\mathrm{L}_{2}(\Psi)}
\end{aligned}
$$

One can easily prove that the weak form (30), with fixed continuous classical boundary optimal control vector $\vec{d} \in \vec{E}$, has a unique "state" solution vector $\vec{z}=\vec{z}_{\vec{d}}$, by applying the same manner employed in the proof of theorem (3). 
Now, by setting once the solution $b_{1}$ in the weak forms of the state equations (11) and once again the solution $\mathrm{b}_{1}+\Delta \mathrm{b}_{1}$, then subtracting the $1^{\text {st }}$ obtained weak form from the other one, we obtain

$$
\begin{aligned}
\mathrm{a}_{1}\left(\Delta \mathrm{b}_{1}, \mathrm{t}_{1}\right)-\left(\Delta \mathrm{b}_{2}+\Delta \mathrm{b}_{3}, \mathrm{t}_{1}\right)_{\mathrm{L}_{2}(\Psi)}+\left(\mathrm{m}_{1}\left(\mathrm{~b}_{1}+\Delta \mathrm{b}_{1}\right)-\mathrm{m}_{1}\left(\mathrm{~b}_{1}\right), \mathrm{t}_{1}\right)_{\mathrm{L}_{2}(\Psi)} \\
=\left(\Delta \mathrm{d}_{1}, \mathrm{t}_{1}\right)_{\mathrm{L}_{2}(\partial \Psi)} \forall \mathrm{t}_{1} \in \mathrm{T}_{1}
\end{aligned}
$$

The same above substituting and subtracting are repeated but from a side with the solutions $b_{2}$ and $b_{2}+\Delta b_{2}$ in the weak form of equation (12) and from thither side with the solutions $b_{3}$ and $b_{3}+\Delta b_{3}$ in the weak form of the state equation (13), respectively, to obtain

$$
\begin{array}{r}
\mathrm{a}_{2}\left(\Delta \mathrm{b}_{2}, \mathrm{t}_{2}\right)+\left(\Delta \mathrm{b}_{1}+\Delta \mathrm{b}_{3}, \mathrm{t}_{2}\right)_{\mathrm{L}_{2}(\Psi)}+\left(\mathrm{m}_{2}\left(\mathrm{~b}_{2}+\Delta \mathrm{b}_{2}\right)-\mathrm{m}_{2}\left(\mathrm{~b}_{2}\right), \mathrm{t}_{2}\right)_{\mathrm{L}_{2}(\Psi)} \\
=\left(\Delta \mathrm{d}_{2}, \mathrm{t}_{2}\right)_{\mathrm{L}_{2}(\partial \Psi)} \forall \mathrm{t}_{2} \in \mathrm{T}_{2} \\
\mathrm{a}_{3}\left(\Delta \mathrm{b}_{3}, \mathrm{t}_{3}\right)+\left(\Delta \mathrm{b}_{1}-\Delta \mathrm{b}_{3}, \mathrm{t}_{3}\right)_{\mathrm{L}_{2}(\Psi)}+\left(\mathrm{m}_{3}\left(\mathrm{~b}_{3}+\Delta \mathrm{b}_{3}\right)-\mathrm{m}_{3}\left(\mathrm{~b}_{3}\right), \mathrm{t}_{3}\right)_{\mathrm{L}_{2}(\Psi)} \\
=\left(\Delta \mathrm{d}_{3}, \mathrm{t}_{3}\right)_{\mathrm{L}_{2}(\partial \Psi)} \forall \mathrm{t}_{3} \in \mathrm{T}_{3}
\end{array}
$$

Adding (31),(32) and (33), then substituting $\overrightarrow{\mathrm{t}}=\left(\mathrm{z}_{1}, \mathrm{z}_{2}, \mathrm{z}_{3}\right)$ in the resulting equation, yield $\mathrm{a}_{1}\left(\Delta \mathrm{b}_{1}, \mathrm{z}_{1}\right)-\left(\Delta \mathrm{b}_{2}+\Delta \mathrm{b}_{3}, \mathrm{z}_{1}\right)_{\mathrm{L}_{2}(\Psi)}+\mathrm{a}_{2}\left(\Delta \mathrm{b}_{2}, \mathrm{z}_{2}\right)+\left(\Delta \mathrm{b}_{1}+\Delta \mathrm{b}_{3}, \mathrm{z}_{2}\right)_{\mathrm{L}_{2}(\Psi)}$

$+\mathrm{a}_{3}\left(\Delta \mathrm{b}_{3}, \mathrm{z}_{3}\right)+\left(\Delta \mathrm{b}_{1}-\Delta \mathrm{b}_{2}, \mathrm{z}_{3}\right)_{\mathrm{L}_{2}(\Psi)}+\left(\left(\mathrm{m}_{1}\left(\mathrm{x}, \mathrm{b}_{1}+\Delta \mathrm{b}_{1}\right), \mathrm{z}_{1}\right)-\mathrm{m}_{1}\left(\mathrm{x}, \mathrm{b}_{1}\right), \mathrm{z}_{1}\right)_{\mathrm{L}_{2}(\Psi)}$

$+\left(\left(\mathrm{m}_{2}\left(\mathrm{x}, \mathrm{b}_{2}+\Delta \mathrm{b}_{2}\right), \mathrm{z}_{2}\right)-\mathrm{m}_{2}\left(\mathrm{x}, \mathrm{b}_{2}\right), \mathrm{z}_{2}\right)_{\mathrm{L}_{2}(\Psi)}+\left(\left(\mathrm{m}_{3}\left(\mathrm{x}, \mathrm{b}_{3}+\Delta \mathrm{b}_{3}\right), \mathrm{z}_{3}\right)-\mathrm{m}_{3}\left(\mathrm{x}, \mathrm{b}_{3}\right), \mathrm{z}_{3}\right)_{\mathrm{L}_{2}(\Psi)}$

$=\left(\Delta \mathrm{d}_{1}, \mathrm{z}_{1}\right)_{\mathrm{L}_{2}(\partial \Psi)}+\left(\Delta \mathrm{d}_{2}, \mathrm{z}_{2}\right)_{\mathrm{L}_{2}(\partial \Psi)}+\left(\Delta \mathrm{d}_{3}, \mathrm{z}_{3}\right)_{\mathrm{L}_{2}(\partial \Psi)}, \forall=\left(\mathrm{z}_{1}, \mathrm{z}_{2}, \mathrm{z}_{3}\right) \in \overrightarrow{\mathrm{V}}$

From the assumptions on $\mathrm{m}_{1}, \mathrm{~m}_{2}, \mathrm{~m}_{3}$ and by using Proposition (2) in reference [19], the Fréchet derivative of $\mathrm{m}_{1}, \mathrm{~m}_{2}, \mathrm{~m}_{3}$ exists. Hence, from Lemma (1) and the Minkowski inequality, (34) becomes

$$
\begin{aligned}
& \mathrm{a}_{1}\left(\Delta \mathrm{b}_{1}, \mathrm{z}_{1}\right)-\left(\Delta \mathrm{b}_{2}+\Delta \mathrm{b}_{3}, \mathrm{z}_{1}\right)_{\mathrm{L}_{2}(\Psi)}+\mathrm{a}_{2}\left(\Delta \mathrm{b}_{2}, \mathrm{z}_{2}\right)+\left(\Delta \mathrm{b}_{1}+\Delta \mathrm{b}_{3}, \mathrm{z}_{2}\right)_{\mathrm{L}_{2}(\Psi)}
\end{aligned}
$$

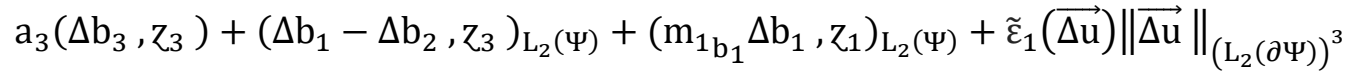

$$
\begin{aligned}
& +\left(\mathrm{m}_{2 \mathrm{~b}_{2}} \Delta \mathrm{b}_{2}, \mathrm{z}_{2}\right)_{\mathrm{L}_{2}(\Psi)}+\tilde{\varepsilon}_{2}(\overrightarrow{\Delta \mathrm{u}})\|\overrightarrow{\Delta \mathrm{u}}\|_{\left(\mathrm{L}_{2}(\partial \Psi)\right)^{3}}+\left(\mathrm{m}_{3 \mathrm{~b}_{3}} \Delta \mathrm{b}_{3}, \mathrm{z}_{3}\right)_{\mathrm{L}_{2}(\Psi)} \\
& +\tilde{\varepsilon}_{3}\left(\overrightarrow{\Delta \mathrm{u})}\|\overrightarrow{\Delta \mathrm{u}}\|_{\left(\mathrm{L}_{2}(\partial \Psi)\right)^{3}}\right. \\
& =\left(\Delta \mathrm{d}_{1}, \mathrm{z}_{1}\right)_{\mathrm{L}_{2}(\partial \Psi)}+\left(\Delta \mathrm{d}_{2}, \mathrm{z}_{2}\right)_{\mathrm{L}_{2}(\partial \Psi)}+\left(\Delta \mathrm{d}_{3}, \mathrm{z}_{3}\right)_{\mathrm{L}_{2}(\partial \Psi)}
\end{aligned}
$$

where $\tilde{\varepsilon}_{1}(\overrightarrow{\Delta \mathrm{d}}), \tilde{\varepsilon}_{2}(\overrightarrow{\Delta \mathrm{d}})$ and $\tilde{\varepsilon}_{3}(\overrightarrow{\Delta \mathrm{d}}) \rightarrow 0$ and $\|\overrightarrow{\Delta \mathrm{d}}\|_{\left(\mathrm{L}_{2}(\partial \Psi)\right)^{3}} \rightarrow 0$ as $\overrightarrow{\Delta \mathrm{d}} \rightarrow 0$.

Subtracting (30) from (35), to get

$$
\begin{gathered}
\left(\mathrm{G}_{01 \mathrm{~b}_{1}}\left(\mathrm{~b}_{1}\right), \Delta \mathrm{b}_{1}\right)_{\mathrm{L}_{2}(\Psi)}+\left(\mathrm{G}_{02 \mathrm{~b}_{2}}\left(\mathrm{~b}_{2}\right), \Delta \mathrm{b}_{2}\right)_{\mathrm{L}_{2}(\Psi)}+\left(\mathrm{G}_{03 \mathrm{~b}_{3}}\left(\mathrm{~b}_{3}\right), \Delta \mathrm{b}_{3}\right)_{\mathrm{L}_{2}(\Psi)} \\
+\widetilde{\varepsilon}_{1}(\overrightarrow{\Delta \mathrm{d}})\|\overrightarrow{\Delta \mathrm{d}}\|_{\left(\mathrm{L}_{2}(\partial \Psi)\right)^{3}}+\widetilde{\varepsilon}_{2}(\overrightarrow{\Delta \mathrm{d}})\|\overrightarrow{\Delta \mathrm{d}}\|_{\left(\mathrm{L}_{2}(\partial \Psi)\right)^{3}}+\tilde{\varepsilon}_{3}(\overrightarrow{\Delta \mathrm{d}})\|\overrightarrow{\Delta \mathrm{d}}\|_{\left(\mathrm{L}_{2}(\partial \Psi)\right)^{3}} \\
=\left(\Delta \mathrm{d}_{1}, \mathrm{Z}_{1}\right)_{\mathrm{L}_{2}(\partial \Psi)}+\left(\Delta \mathrm{d}_{2}, \mathrm{Z}_{2}\right)_{\mathrm{L}_{2}(\partial \Psi)}+\left(\Delta \mathrm{d}_{3}, \mathrm{z}_{3}\right)_{\mathrm{L}_{2}(\partial \Psi)}
\end{gathered}
$$

Now, from the assumptions on $\mathbf{t}_{01}, \mathbf{T}_{02}, \mathbf{t}_{03}, \mathbf{t}_{04}, \mathbf{t}_{05}, \mathbf{t}_{06}$, Proposition (2) in reference [19], and then using the result of Lemma (1), we have

$$
\begin{aligned}
\mathrm{T}_{0}(\overrightarrow{\mathrm{d}}+\overrightarrow{\Delta \mathrm{d}})-\mathrm{T}_{0}(\overrightarrow{\mathrm{d}})= & \iint_{\Psi}\left(\mathrm{T}_{01 \mathrm{~b}_{1}}\left(\mathrm{x}, \mathrm{b}_{1}\right) \Delta \mathrm{b}_{1}+\mathrm{T}_{02 \mathrm{~b}_{2}}\left(\mathrm{x}, \mathrm{b}_{2}\right) \Delta \mathrm{b}_{2}\right. \\
& \left.+\mathrm{G}_{03 \mathrm{~b}_{3}}\left(\mathrm{x}, \mathrm{b}_{3}\right) \Delta \mathrm{b}_{3}\right) \mathrm{dx}_{1} \mathrm{dx}_{2} \\
& +\int_{\partial \Psi}\left(\mathrm{G}_{04 \mathrm{~d}_{1}}\left(\mathrm{x}, \mathrm{d}_{1}\right) \Delta \mathrm{d}_{1}+\mathrm{T}_{05 \mathrm{~d}_{2}}\left(\mathrm{x}, \mathrm{d}_{2}\right) \Delta \mathrm{d}_{2}\right. \\
& \left.+\mathrm{G}_{06 \mathrm{~d}_{3}}\left(\mathrm{x}, \mathrm{d}_{3}\right) \Delta \mathrm{d}_{3}\right) \mathrm{d} \gamma+\varepsilon_{4}(\overrightarrow{\Delta \mathrm{d}})\|\overrightarrow{\Delta \mathrm{d}}\|_{\left(\mathrm{L}_{2}(\Psi)\right)^{3}}
\end{aligned}
$$

where $\varepsilon_{4}(\overrightarrow{\Delta \mathrm{d}}) \rightarrow 0$, as $\overrightarrow{\Delta \mathrm{d}} \rightarrow 0$

By substituting (36) in the above equality, we get

$$
\begin{aligned}
& \mathrm{T}_{0}(\overrightarrow{\mathrm{d}}+\overrightarrow{\Delta \mathrm{d}})-\mathrm{T}_{0}(\overrightarrow{\mathrm{d}})=\int_{\partial \Psi}\left(\mathrm{z}_{1}, \mathrm{t}_{04 \mathrm{~d}_{1}}\right) \Delta \mathrm{d}_{1} \mathrm{~d} \gamma+\int_{\partial \Psi}\left(\mathrm{z}_{2}, \mathrm{t}_{05 \mathrm{~d}_{2}}\right) \Delta \mathrm{d}_{2} \mathrm{~d} \gamma \\
& +\int_{\partial \Psi}\left(\mathrm{z}_{3}, \mathrm{G}_{06 \mathrm{~d}_{3}}\right) \Delta \mathrm{d}_{3} \mathrm{~d} \gamma+\tilde{\varepsilon}_{5}(\overrightarrow{\Delta \mathrm{d}})\|\overrightarrow{\Delta \mathrm{d}}\|_{\left(\mathrm{L}_{2}(\partial \Psi)\right)^{3}}
\end{aligned}
$$

where

$\tilde{\varepsilon}_{5}(\overrightarrow{\Delta \mathrm{d}})=\tilde{\varepsilon}_{4}(\overrightarrow{\Delta \mathrm{d}})-\tilde{\varepsilon}_{1}(\overrightarrow{\Delta \mathrm{d}})-\tilde{\varepsilon}_{2}(\overrightarrow{\Delta \mathrm{d}})-\tilde{\varepsilon}_{3}(\overrightarrow{\Delta \mathrm{d}}) \rightarrow 0$, as $\overrightarrow{\Delta \mathrm{d}} \rightarrow 0$.

But from the definition of the Fréchet derivative of $\mathrm{T}_{0}$, one gets

$$
\overrightarrow{\mathrm{U}_{0}^{\prime}}(\overrightarrow{\mathrm{d}}) \overrightarrow{\Delta \mathrm{d}}=\int_{\partial \Psi} U_{\overrightarrow{\mathrm{d}}}^{\mathrm{T}} \overrightarrow{\Delta \mathrm{d}} \mathrm{d} \gamma \text {, where }{U_{\overrightarrow{\mathrm{d}}}^{\mathrm{T}}}^{\mathrm{T}} \text { is defined above. }
$$

Note: In the proof of the above theorem, we have found the Fréchet derivative for the functional $\mathrm{T}_{0}$, so the same technique is used to find the Fréchet derivative for $\mathrm{G}_{1}$ and $\mathrm{T}_{2}$. 


\section{Theorem (4):}

(a) If assumptions (I), (II), and (III) are held, then $\overrightarrow{\mathrm{E}}$ is convex, and if $\overrightarrow{\mathrm{d}} \in \overrightarrow{\mathrm{E}}_{\mathrm{A}}$ is a continuous classical boundary optimal control vector, then $\forall \mathrm{P}=0,1,2$ and there exist multipliers $\xi_{\mathrm{P}} \in \mathbb{R}$, with $\xi_{0} \geq 0, \xi_{2} \geq 0 \sum_{\mathrm{P}=1}^{2}\left|\xi_{\mathrm{P}}\right|=1$, so that the following Kuhn-Tucker- Lagrange's Multipliers conditions are held:

$$
\begin{gathered}
\int_{\partial \Psi} H_{\overrightarrow{\mathrm{d}}}^{\mathrm{T}} \cdot \overrightarrow{\Delta \mathrm{d}} \mathrm{d} \gamma \geq 0, \text { with } \overrightarrow{\Delta \mathrm{d}}=\overrightarrow{\mathrm{w}}-\overrightarrow{\mathrm{d}}, \forall \overrightarrow{\mathrm{e}} \in \overrightarrow{\mathrm{E}} \\
\text { wheret }_{\sigma \mathrm{dj}}=\sum_{\mathrm{P}=0}^{2} \xi_{\mathrm{P}} \mathrm{G}_{\sigma \mathrm{dj}} \& z_{\mathrm{j}}=\sum_{\mathrm{P}=0}^{2} \xi_{\mathrm{P}} Z_{\mathrm{Pj}},(\text { for } \mathrm{j}=0,1,2, \sigma=4,5,6) \text { in (Theorem (5)), } \\
\xi_{2} \mathrm{G}_{2}(\overrightarrow{\mathrm{d}})=0, \text { (Transversality conditions) }
\end{gathered}
$$

(b) (Minimum Principle in point wise weak form): The inequality (39a) is equivalent to

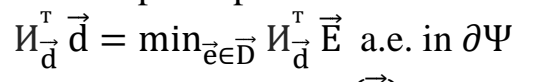

Proof: (a) from Theorem (3) $, \mathrm{G}_{\mathrm{P}}(\overrightarrow{\mathrm{d}}) \forall \mathrm{P}=0,1,2$ and at any $\overrightarrow{\mathrm{d}} \in \overrightarrow{\mathrm{E}}$ ) has a continuous Fréchet derivative. Since the continuous classical boundary optimal control vector $\vec{d} \in \vec{E}_{A}$ is optimal, then by using the Kuhn-Tucker- Lagrange's Multipliers theorem $\forall \mathrm{P}=0,1,2$, there exist multipliers $\xi_{\mathrm{P}} \in \mathbb{R}$ with $\xi_{0} \geq 0, \xi_{2} \geq 0, \sum_{\ell=1}^{2}\left|\xi_{P}\right|=1$, such that

$$
\begin{aligned}
& \left(\sum_{\mathrm{P}=0}^{2} \xi_{\mathrm{P}} \overrightarrow{\mathrm{T}_{\mathrm{P}}^{\prime}}(\overrightarrow{\mathrm{d}})\right)_{\mathrm{L}_{2}(\partial \Psi)} \cdot(\overrightarrow{\mathrm{e}}-\overrightarrow{\mathrm{d}}) \geq 0, \forall \overrightarrow{\mathrm{e}} \in \overrightarrow{\mathrm{E}} \\
& \xi_{2} \mathrm{~T}_{2}(\overrightarrow{\mathrm{d}})=0
\end{aligned}
$$

Then, from Theorem (3), (41a) with the setting $\Delta \mathrm{d}_{1}=\mathrm{e}_{1}-\mathrm{d}_{1}, \Delta \mathrm{d}_{2}=\mathrm{e}_{2}-\mathrm{d}_{2}, \Delta \mathrm{d}_{3}=\mathrm{e}_{3}-\mathrm{d}_{3}$, we can rewrite $\forall \overrightarrow{\mathrm{e}} \in \overrightarrow{\mathrm{E}}$ as

$\int_{\partial \Psi}\left[\left(\mathrm{z}_{1}+\mathrm{T}_{4 \mathrm{~d}_{1}}\right) \Delta \mathrm{d}_{1}+\left(\mathrm{z}_{2}+\mathrm{G}_{5 \mathrm{~d}_{2}}\right) \Delta \mathrm{d}_{2}+\left(\mathrm{z}_{3}+\mathrm{G}_{6 \mathrm{~d}_{3}}\right) \Delta \mathrm{d}_{3}\right] \mathrm{d} \gamma \geq 0$

where $\mathrm{Z}_{\mathrm{j}}=\sum_{\mathrm{P}=0}^{2} \xi_{\mathrm{P}} \mathrm{Z}_{\mathrm{jP}}, \mathrm{T}_{\sigma \mathrm{dj}}=\sum_{\mathrm{P}=0}^{2} \xi_{\mathrm{P}} \mathrm{G}_{\sigma \mathrm{dj}}$, for $\mathrm{j}=1,2,3, \sigma=4,5,6$

$\Rightarrow \int_{\partial \Psi} U_{\overrightarrow{\mathrm{d}}}^{\mathrm{T}} \cdot \overrightarrow{\Delta \mathrm{d}} \mathrm{d} \gamma \geq 0, \forall \overrightarrow{\mathrm{e}} \in \overrightarrow{\mathrm{E}}, \overrightarrow{\Delta \mathrm{d}}=\overrightarrow{\mathrm{e}}-\overrightarrow{\mathrm{d}}$.

(b) Let $\left\{\overrightarrow{\mathrm{d}}_{\mathrm{n}}\right\}$ be a sequence, dense in $\overrightarrow{\mathrm{E}}_{\overrightarrow{\mathrm{D}}}$, and $\mathrm{S} \subset \partial \Psi$ be a measurable set, such that

$$
\vec{E}(x)=\left\{\begin{array}{l}
\vec{d}_{n}(x), \text { for } x \text { belong in } S \\
\vec{d}(x), \text { for } x \text { not belong in } S
\end{array}\right.
$$

Hence (41a), becomes

$\int_{S} U_{\vec{d}}^{T} \cdot\left(\vec{d}_{n}-\vec{d}\right) d s \geq 0$, for any $S \subset \partial \Psi$. Then, by using Theorem (2) in reference [19], we obtain $U_{\overrightarrow{\mathrm{d}}} \cdot\left(\overrightarrow{\mathrm{d}}_{\mathrm{n}}-\overrightarrow{\mathrm{d}}\right) \geq 0$, a.e on $\partial \Psi$.

The above inequality satisfies on $\partial \Psi$, except in a subset $\partial \Psi_{n}$ with $\tau\left(\partial \Psi_{n}\right)=0$, for each $n$, where $\tau$ is a Lebesgue measure, then this equality holds on $\partial \Psi$ except in $U_{n} \partial \Psi_{n}$ with $\tau\left(U_{n} \partial \Psi_{n}\right)=0$. But $\left\{\vec{d}_{n}\right\}$ is a dense in $\vec{E}$, then there exists $\vec{d} \in \vec{E}$, such that

$U_{\vec{d}}^{\mathrm{T}} \overrightarrow{\mathrm{d}}=\min _{\overrightarrow{\mathrm{e}} \in \overrightarrow{\mathrm{D}}} U_{\overrightarrow{\mathrm{d}}}^{\mathrm{T}} \overrightarrow{\mathrm{e}}$, a.e, on $\partial \Psi$.

\section{The Sufficient Conditions for Optimality of the Continuous Classical Boundary Optimal Control Vector}

Theorem (7): In addition to assumptions (I), (II), and (III), if $\mathrm{m}_{1}, \mathrm{~m}_{2}, \mathrm{~m}_{3}, \mathrm{t}_{11}, \mathrm{t}_{12}, \mathrm{t}_{13}$ are affine with respect to $\vec{b}, \mathbf{G}_{14}, \mathrm{G}_{15}, \mathrm{G}_{16}$ are affine with respect to $\overrightarrow{\mathrm{d}}, \mathrm{5}_{1}, \mathrm{5}_{2}, \mathrm{5}_{3}$ are bounded functions for $\mathrm{x}$. Also, if $\mathrm{g}_{\mathrm{P}_{\sigma}}(\mathrm{P}=0,2, \sigma=1,2,3,4,5,6)$ are convex with respect to $\mathrm{b}_{1}, \mathrm{~b}_{2}, \mathrm{~b}_{3}, \mathrm{~d}_{1}, \mathrm{~d}_{2}, \mathrm{~d}_{3}$, respectively, for each $\mathrm{x}$, then the necessary and sufficient conditions for optimality in the previous theorem (6), with $\xi_{0}>0$, are sufficient .

Proof: Assume that $\overrightarrow{\mathrm{d}} \in \overrightarrow{\mathrm{E}}_{\mathrm{A}}, \overrightarrow{\mathrm{d}}$ satisfies the conditions (39a) and (39b).

Let

$$
\begin{aligned}
\mathrm{T}(\overrightarrow{\mathrm{d}})=\sum_{\mathrm{P}=0}^{2} \xi_{\mathrm{P}} \mathrm{G}(\overrightarrow{\mathrm{d}}) \Rightarrow \overrightarrow{\mathrm{G}_{0}^{\prime}}(\overrightarrow{\mathrm{d}}) \overrightarrow{\Delta \mathrm{d}}=\sum_{\mathrm{P}=0}^{2} \xi_{\mathrm{P}} \int_{\partial \Psi}\left[\left(\left(\mathrm{Z}_{\mathrm{P} 1}+\mathrm{T}_{\mathrm{P}_{4} \mathrm{~d}_{1}}\right) \Delta \mathrm{d}_{1}+\left(\mathrm{ZP} 2_{\mathrm{P} 2}+\mathrm{G}_{\mathrm{P} 5 \mathrm{~d}_{2}}\right) \Delta \mathrm{d}_{2}\right.\right. \\
\left.\left.+\left(\mathrm{z}_{\mathrm{P} 3}+\mathrm{T}_{\mathrm{P} 6 \mathrm{~d}_{3}}\right) \Delta \mathrm{d}_{3}\right)\right] \mathrm{d} \gamma \\
=\int_{\partial \Psi} \mathrm{h}_{\overrightarrow{\mathrm{d}}}\left(\mathrm{x}, \mathrm{z}_{1}, \mathrm{z}_{2}, \mathrm{z}_{3}, \mathrm{~d}_{1}, \mathrm{~d}_{2}, \mathrm{~d}_{3}\right) \cdot \overrightarrow{\Delta \mathrm{d}} \mathrm{d} \gamma \geq 0 .
\end{aligned}
$$

and

$\mathrm{m}_{1}\left(\mathrm{x}, \mathrm{b}_{1}\right)=\mathrm{m}_{11}(\mathrm{x}) \mathrm{b}_{1}+\mathrm{m}_{12}(\mathrm{x}), \mathrm{m}_{2}\left(\mathrm{x}, \mathrm{b}_{2}\right)=\mathrm{m}_{21}(\mathrm{x}) \mathrm{b}_{2}+\mathrm{m}_{22}(\mathrm{x})$, 
$\mathrm{m}_{3}\left(\mathrm{x}, \mathrm{b}_{3}\right)=\mathrm{m}_{31}(\mathrm{x}) \mathrm{b}_{1}+\mathrm{m}_{32}(\mathrm{x})$, and $\mathrm{h}_{\sigma}(\mathrm{x})$ are bounded for $\sigma=1,2,3, \forall \mathrm{x} \in \Psi$.

Let $\overrightarrow{\mathrm{d}}=\left(\mathrm{d}_{1}, \mathrm{~d}_{2}, \mathrm{~d}_{3}\right), \overrightarrow{\overrightarrow{\mathrm{d}}}=\left(\overline{\mathrm{d}}_{1}, \overline{\mathrm{d}}_{2}, \overline{\mathrm{d}}_{3}\right)$ be given two continuous classical boundary optimal control vectors, then the corresponding "state" solution vector are $\vec{b}=\left(b_{1}, b_{2}, b_{3}\right), \vec{b}=\left(\bar{b}_{1}, \bar{b}_{2}, \bar{b}_{3}\right)$ By substituting $(\vec{b}, \vec{d})$ in (1)-(6) and multiplying the resulting equations by $\theta \in[0,1]$ once, we again the substitution of the pair $(\overrightarrow{\bar{b}}, \overrightarrow{\bar{d}})$ in (1)-(6). By multiplying the result by $\bar{\theta}=(1-\theta)$, and finally summing each pair from the corresponding equations together, we get:

$$
\begin{aligned}
& \mathrm{A}_{1}\left(\theta \mathrm{b}_{1}+\bar{\theta} \overline{\mathrm{b}}_{1}\right)+\left(\theta \mathrm{b}_{1}+\bar{\theta} \overline{\mathrm{b}}_{1}\right)-\left(\theta \mathrm{b}_{2}+\bar{\theta} \overline{\mathrm{b}}_{2}\right)-\left(\theta \mathrm{b}_{3}+\bar{\theta} \overline{\mathrm{b}}_{3}\right)+\mathrm{m}_{11}(\mathrm{x})\left(\theta \mathrm{b}_{1}+\bar{\theta} \overline{\mathrm{b}}_{1}\right) \\
& +\mathrm{m}_{12}(\mathrm{x})=\mathrm{H}_{1}(\mathrm{x}) \\
& \sum_{\mathrm{i}, \mathrm{j}=0}^{2} \mathrm{a}_{1 \mathrm{ij}} \frac{\partial}{\partial \mathrm{n}_{1}}\left(\theta \mathrm{b}_{1}+\bar{\theta} \overline{\mathrm{b}}_{1}\right)=\theta \mathrm{d}_{1}+\bar{\theta} \overline{\mathrm{d}}_{1} \\
& \mathrm{~A}_{2}\left(\theta \mathrm{b}_{2}+\bar{\theta} \overline{\mathrm{b}}_{2}\right)+\left(\theta \mathrm{b}_{1}+\bar{\theta} \overline{\mathrm{b}}_{1}\right)+\left(\theta \mathrm{b}_{2}+\bar{\theta} \overline{\mathrm{b}}_{2}\right)+\left(\theta \mathrm{b}_{3}+\bar{\theta} \overline{\mathrm{b}}_{3}\right)+\mathrm{m}_{21}(\mathrm{x})\left(\theta \mathrm{b}_{2}+\bar{\theta} \overline{\mathrm{b}}_{2}\right) \\
& +\mathrm{m}_{22}(\mathrm{x})=\mathrm{K}_{2}(\mathrm{x}) \\
& \sum_{\mathrm{i}, \mathrm{j}=0}^{2} \mathrm{a}_{2 \mathrm{ij}} \frac{\partial}{\partial \mathrm{n}_{2}}\left(\theta \mathrm{b}_{2}+\bar{\theta} \overline{\mathrm{b}}_{2}\right)=\theta \mathrm{u}_{2}+\bar{\theta} \overline{\mathrm{d}}_{2}
\end{aligned}
$$

and

$$
\begin{gathered}
\mathrm{A}_{3}\left(\theta \mathrm{b}_{3}+\bar{\theta} \overline{\mathrm{b}}_{3}\right)+\left(\theta \mathrm{b}_{1}+\bar{\theta} \overline{\mathrm{b}}_{1}\right)-\left(\theta \mathrm{b}_{2}+\bar{\theta} \overline{\mathrm{b}}_{2}\right)+\left(\theta \mathrm{b}_{3}+\bar{\theta} \overline{\mathrm{b}}_{3}\right)+\mathrm{m}_{31}(\mathrm{x})\left(\theta \mathrm{b}_{3}+\bar{\theta} \overline{\mathrm{b}}_{3}\right) \\
+\mathrm{m}_{32}(\mathrm{x})=\mathrm{F}_{3}(\mathrm{x}) \\
\sum_{\mathrm{i}, \mathrm{j}=0}^{2} \mathrm{a}_{3 \mathrm{ij}} \frac{\partial}{\partial \mathrm{n}_{3}}\left(\theta \mathrm{b}_{3}+\bar{\theta} \overline{\mathrm{b}}_{3}\right)=\theta \mathrm{d}_{3}+\bar{\theta} \overline{\mathrm{d}}_{3}
\end{gathered}
$$

Now, if we have the continuous classical boundary optimal control vector $\overrightarrow{\overline{\mathrm{d}}}=\left(\overline{\overline{\mathrm{d}}}_{1}, \overline{\overline{\mathrm{d}}}_{2}, \overline{\overline{\mathrm{d}}}_{3}\right)$ with $\overline{\overline{\mathrm{d}}}_{1}=$ $\theta \mathrm{u}_{1}+\tilde{\alpha} \overline{\mathrm{d}}_{1}, \overline{\mathrm{d}}_{2}=\theta \mathrm{u}_{2}+\bar{\theta} \overline{\mathrm{d}}_{2}$ and $\overline{\mathrm{d}}_{3}=\theta \mathrm{u}_{3}+\tilde{\alpha} \overline{\mathrm{d}}_{3}$. Then, from (42a\&b), (43a\&b), and (44a\&b), one gets that the "state" solution vector $\left(\overline{\bar{b}}_{1}=b_{1} \overline{\bar{d}}_{1}, \overline{\bar{b}}_{2}=b_{2} \overline{\overline{\mathrm{d}}}_{2}, \overline{\overline{\mathrm{b}}}_{3}=\mathrm{b}_{3} \overline{\overline{\mathrm{d}}}_{3}\right)$ with $\overline{\bar{b}}_{1}=\theta \mathrm{b}_{1_{\mathrm{u}_{1}}}+$ $\bar{\theta} \mathrm{b}_{1 \overline{\mathrm{d}}_{1}}=\theta \mathrm{b}_{1}+\bar{\theta} \overline{\mathrm{b}}_{1} \overline{\overline{\mathrm{b}}}_{2}=\theta \mathrm{b}_{2_{\mathrm{u}_{2}}}+\bar{\theta} \mathrm{b}_{2 \overline{\mathrm{d}}_{2}}=\theta \mathrm{b}_{2}+\bar{\theta} \overline{\mathrm{b}}_{2}$ and $\overline{\overline{\mathrm{b}}}_{3}=\theta \mathrm{b}_{\mathrm{u}_{3}}+\bar{\theta} \mathrm{b}_{2 \overline{\mathrm{d}}_{3}}=\theta \mathrm{b}_{3}+\bar{\theta} \overline{\mathrm{b}}_{3}$ are their corresponding solution, i.e. they satisfy (1-6), respectively. So, the operators $d_{1} \mapsto b_{1_{d_{1}}}$, $d_{2} \mapsto b_{d_{2}}$ and $d_{3} \mapsto b_{3_{d_{3}}}$ are convex-linear with respect to $\left(b_{1}, d_{1}\right),\left(b_{2}, d_{2}\right)$ and $\left(b_{3}, d_{3}\right)$, respectively.

Now, from this result and since $\mathrm{G}_{11}, \mathrm{G}_{12}, \mathrm{G}_{13}, \mathrm{G}_{14}, \mathrm{G}_{15}, \mathrm{G}_{16}$ are affine with respect to $\mathrm{b}_{1}, \mathrm{~b}_{2}, \mathrm{~b}_{3}, \mathrm{~d}_{1}, \mathrm{~d}_{2}, \mathrm{~d}_{3}$, respectively, on $\Psi$, we get that $\forall \mathrm{x} \in \Psi, \mathrm{T}_{\mathrm{P}}(\overrightarrow{\mathrm{d}})$ is convex-linear in $(\overrightarrow{\mathrm{b}}, \overrightarrow{\mathrm{d}})$. Also, since $(\forall \mathrm{P}=0,2 \& \forall \mathrm{x} \in \Psi) \mathrm{G}_{\mathrm{P} 1}, \mathrm{~T}_{\mathrm{P} 2}, \mathrm{~T}_{\mathrm{P} 3}, \mathrm{~T}_{\mathrm{P} 4}, \mathrm{~T}_{\mathrm{P} 5}, \mathrm{G}_{\mathrm{P} 6}$ are convex with respect to $\mathrm{b}_{1}, \mathrm{~b}_{2}, \mathrm{~b}_{3}$, $d_{1}, d_{2}$ and $d_{3}$ respectively, i.e. $T(\vec{d})$ is convex with respect to $\vec{b}$ and $\vec{d}$, then $\mathrm{G}(\vec{d})$ is convex in $\vec{b}$ and $\overrightarrow{\mathrm{d}}$ i $\mathrm{n}$ the convex set $\overrightarrow{\mathrm{E}}$ and has a continuous Fréchet derivative that satisfies $\overrightarrow{\mathrm{G}_{0}^{\prime}}(\overrightarrow{\mathrm{d}}) \cdot \overrightarrow{\Delta \mathrm{d}} \geq 0 \Rightarrow \mathrm{G}(\overrightarrow{\mathrm{d}})$ has a minimum at $\overrightarrow{\mathrm{d}}$, i.e. $\mathrm{T}(\overrightarrow{\mathrm{d}}) \leq \mathrm{T}(\overrightarrow{\mathrm{w}}), \forall \overrightarrow{\mathrm{e}} \in \overrightarrow{\mathrm{E}}$, then we have

$$
\sum_{\mathrm{P}=0}^{2} \xi_{\mathrm{P}} \mathrm{T}(\overrightarrow{\mathrm{d}}) \leq \sum_{\mathrm{P}=0}^{2} \xi_{\mathrm{P}} \mathrm{T}(\overrightarrow{\mathrm{e}})
$$

Now, let $\vec{E}$ be also admissible and satisfies the Transversality condition, then (45) becomes $\mathrm{T}_{0}(\overrightarrow{\mathrm{d}}) \leq$ $\mathrm{T}_{0}(\overrightarrow{\mathrm{e}}), \forall \overrightarrow{\mathrm{e}} \in \overrightarrow{\mathrm{E}}$, i.e.

$\overrightarrow{\mathrm{d}}$ is a classical continuous boundary control vector problem .

\section{Conclusions}

The existence and uniqueness theorem for the "state" solution vector of the triple nonlinear partial differential equations of elliptic type is proved successfully, when the classical continuous boundary control vector is given. The proof of the existence of the classical continuous boundary control vector, ruling by the considered triple nonlinear partial differential equations of elliptic type, is demonstrated with the equality and inequality constraints. The studying of the existence solution of the triple adjoint equations related with the triple nonlinear partial differential equations of elliptic type is demonstrated with the equality and inequality constraints. Finally, the theorems of both the necessary and sufficient conditions for optimality of the triple nonlinear partial differential equations of elliptic type, through the Kuhn-Tucker-Lagrange's Multiplires with equality and inequality constraints, is demonstrated. 


\section{References}

1. Grigorenko, N. L., Grigorieva, È. V., Roi, P. K., and Khailov, E. N. 2019. Optimal control problems for a mathematical model of the treatment of psoriasis. Computational mathimatics and modeling, 30(4): 352-363.

2. Kahina, L., Spiteri, P., Demim, F., Mohamed, A., Nemra, A., and Messine, F. 2018 Application optimal control for a problem aircraft flight. Journal of engineering science and technology review, 11(1): 156-164.

3. Aderinto, Y. O., Afolabi, A. O., and Issa, I. T. 2017. On optimal planning of electric power generation systems. Journal of mathematics, 50(1): 89-95.

4. Kryazhimskii, A. V., and Taras'ev, A. M. 2016. Optimal control for proportional economic growth. Pleiades publishing. Ltd, 293(1): S101-S119.

5. Afshar, M., Merrikh-Bayat, F., and Razvan, M. R. 2016. Stepwise solution for optimal Control problems. Journal of science and engineering, 13(2): 024-037.

6. Mabonzo,V. D., Ampini, D. 2019. Existence of optimal control for a nonlinear partial Differential equation of hyperbolic-type. European Journal of Pure And Applied Mathematics, 12(4):15951601.

7. Kadhem, G.M. 2015. The continuous classical optimal control problem of partial differential equations. M.Sc. Thesis, Department of mathematics, College of Science, Mustansiriyah University, Iraq.

8. Al-Rawdanee, E.H.M. 2015. The continuous classical optimal control problem of a non-Linear partial differential equations of elliptic type. M.Sc. Thesis, Department of mathematics, College of Science, Mustansiriyah University, Iraq.

9. Al-Hawasy, J. 2019. The continuous classical boundary optimal control of couple nonlinear hyperbolic boundary value problem with equality and inequality constraints. Baghdad science journal, 16(4): 1064-1074.

10. Kadhem, G.M. 2015. The continuous classical optimal control problem of partial Differential equations. M.Sc. Thesis, Department of mathematics, College of Science, Mustansiriyah University, Iraq.

11. Al-Hawasy, J. and Al-Qaisi, S. 2019. The solvability of the continuous classical Boundary optimal control of couple nonlinear elliptic partial differential equations with State constraints. AlMustansiriyah journal of science, 30(1):143-151.

12. Al-Hawasy, J. and Naeif, A. 2017. The continuous classical boundary optimal control of A couple nonlinear parabolic partial differential equations. $1^{\text {st }}$ Scientific international conference, Special Issus: 123-136.

13. Al-Hawasy, J. and Ali, Lamyaa 2020. Constraints Optimal Control Governing by Triple Nonlinear Hyperbolic Boundary Value Problem. Journal of Applied Mathematics, Hindawi, 2020: 1-14.

14. Al-Hawasy, J. and Jaber, M. 2020 The continuous classical optimal control governing by triple parabolic boundary value problem. Ibn Al-Haitham for Pure and Applied Science, 33(1): 129-142.

15. Al-Hawasy, J. and Jasim, D. 2020. The continuous classical optimal control problems of a triple elliptic partial differential equations. Ibn Al-Haitham for pure and applied science, 33(1):143-151.

16. Al-Hawasy, J. 2019. Solvability for continuous classical optimal control associated with triple hyperbolic boundary value problem. Accepted in Ijpam,

17. Al-Hawasy, J. and Jaber, M. 2019. The continuous classical boundary optimal control Vector governing by triple linear partial differential equations of parabolic type. Accepted in $\mathrm{Ibn} \mathrm{Al}$ Haitham for pure and applied science.

18. Borzabadi, A. H., Kamyad, A. V., and Farahi, M. H. G. 2 Optimal Control of the Heat Equation in an Inhomogeneous Body. J. Appl. Math. and Computing, 15(1-2): 127-146

19. Chryssoverghi, I. and Bacopoulos, A. 1993. Approximation of Relaxed Nonlinear Parabolic Optimal Control Problems. Journal of Optimization Theory and Approximations, 77(1).

20. Bacopoulos, A. and Chryssoverghi, I. 2003. Numerical Solutions of Partial Differential Equations by Finite Elements Methods. Symeom Publishing Co, Athens.

21. Brezis, H. 2011. Functional Analysis, Sobolev Spaces and Partial Differential Equations. Springer, New York-USA 